\title{
Optimal Tolerance Design and Optimization for a Pharmaceutical Quality Characteristic
}

\author{
Seong Hoon Jeong, ${ }^{1}$ Pauline Kongsuwan, ${ }^{2}$ Nguyen Khoa Viet Truong, ${ }^{2}$ and Sangmun Shin ${ }^{3}$ \\ ${ }^{1}$ College of Pharmacy, Dongguk University-Seoul, Goyang 410-820, Republic of Korea \\ ${ }^{2}$ Department of Information and Communication Systems, Inje University, Gimhae 621-749, Republic of Korea \\ ${ }^{3}$ Department of Industrial \& Systems Management Engineering, Dong-A University, Busan 604-714, Republic of Korea
}

Correspondence should be addressed to Sangmun Shin; sshin@dau.ac.kr

Received 7 November 2012; Revised 18 January 2013; Accepted 19 January 2013

Academic Editor: Ming Li

Copyright (C) 2013 Seong Hoon Jeong et al. This is an open access article distributed under the Creative Commons Attribution License, which permits unrestricted use, distribution, and reproduction in any medium, provided the original work is properly cited.

\begin{abstract}
A number of pharmaceutical quality characteristics are destructive or too costly to inspect. However, most quality improvement tools developed in the pharmaceutical research community typically assume that quality characteristics are nondestructive. This paper proposes a new design system for quality improvement by incorporating the concept of surrogate variables with the concepts of robust design (RD) and tolerance design (TD). The proposed robust-tolerance design paradigm determines the optimal factor setting and specification limits simultaneously, thereby improving quality of pharmaceutical products. In addition, the proposed methodology can provide the optimal tolerance as a mathematical closed-form solution. Finally, a numerical example and its associated sensitivity analysis for a pharmaceutical case are conducted for verification purposes. Based on the numerical example results, the proposed approach could provide robust factor settings with significant tradeoffs between quality and cost.
\end{abstract}

\section{Introduction}

Effectively considering product quality in the early stage of product/process design could reduce production cost, which is one of the key issues in quality engineering. It has been more than twenty years that enterprises integrate product quality into their business strategies. In off-line quality improvement inspired by Taguchi $[1,2]$, there are three major phases as follows:

(i) system design: a design phase for an activity of proceeding and functional prototyping from an identified set of requirements,

(ii) robust design ( $\mathrm{RD})$ : a design phase for determining the optimal factor setting which minimizes product/process variability and bias,

(iii) tolerance design (TD): a design phase for determining optimal specification limits in order to improve product quality by reducing the variability of quality characteristics.
$\mathrm{RD}$ directly reflects a design for an optimal factor setting [3] with the aims of minimizing process bias and variability of a quality characteristic. Because of its practicability in reducing inherent uncertainty associated with design factors and system performance, the applications of $\mathrm{RD}$ techniques have resulted in significant improvements of product quality, manufacturability, and reliability at low cost. Even though the ad hoc robust design methods suggested by Taguchi remain controversial due to various mathematical flaws, there is little disagreement among researchers and practitioners about his basic philosophy. The controversy surrounding Taguchi's assumptions, experimental design, and statistical analysis has been well addressed by Box [4], Box et al. [5], León et al. [6], Nair [7], and Tsui [8]. Consequently, researchers have closely examined alternative approaches using wellestablished statistical and optimization tools. As an alternative for modeling process relationships, Vining and Myers [9] introduced a dual response approach based on response surface methodology (RSM) by separately estimating the response functions of process mean and variance, thereby achieving the primary goal of robust design by minimizing 


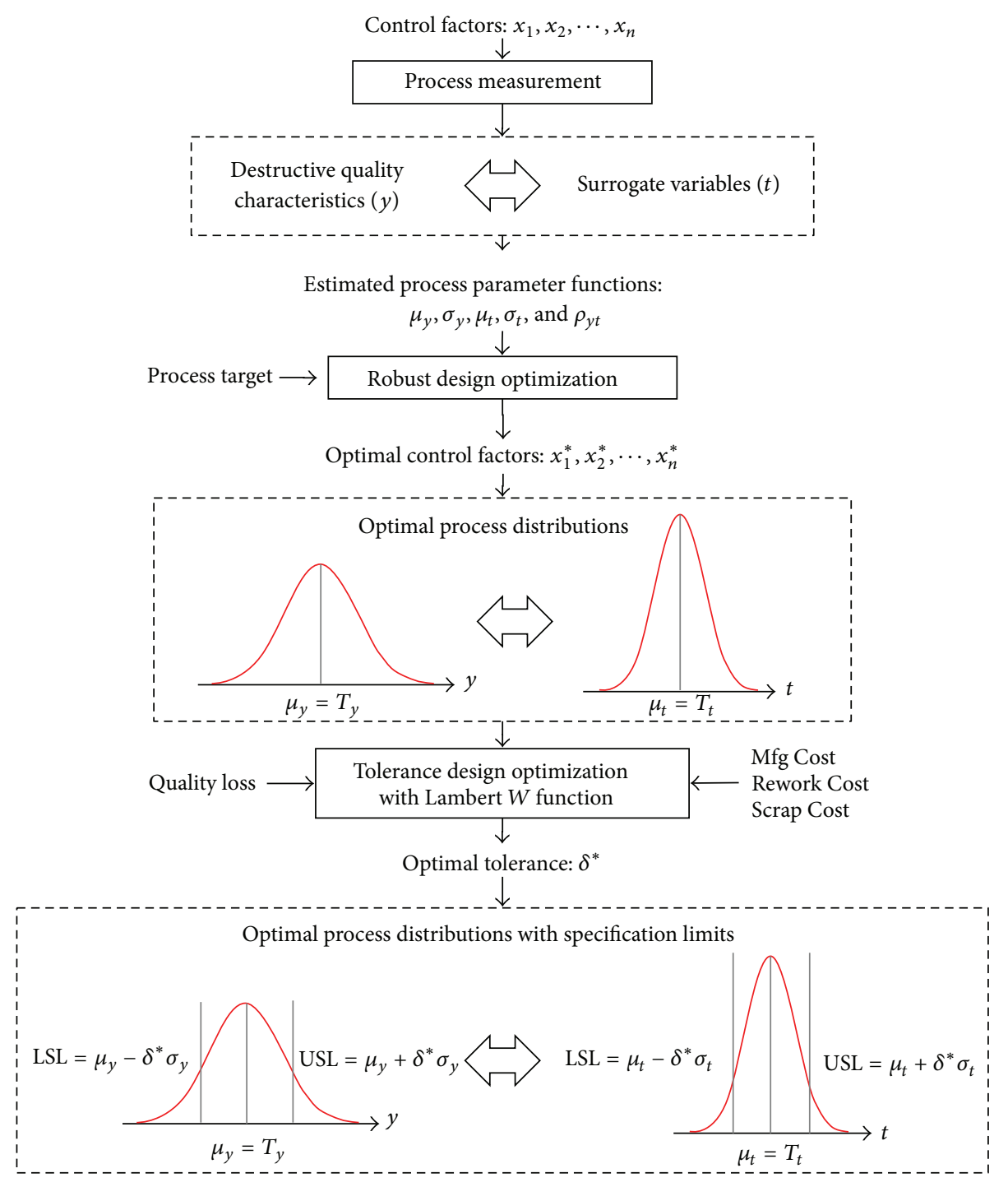

FIGURE 1: An overview of the proposed robust-tolerance design approach.

the process variance while adjusting the process mean at the target. Many extensions of the dual response approach are reported in the literature, such as in [3,10-16].

As advanced types of automated inspection equipment become an integral part of modern manufacturing systems, the implementation of a tolerance on every product item has increased in recent years and become an attractive means of manufacturing high quality products. Automated equipment can perform rigorous inspection procedures and provide a consistent performance. When the nature of a performance variable is destructive, a surrogate variable, which is correlated to the performance variable, can be used effectively for the TD optimization under $100 \%$ inspections in many industrial applications. K. Tang and J. Tang [17] provided an excellent discussion of the overall concept for determining the optimal product specification limits under various screening inspection environments including the Deming's all-or-none rules, Taguchi's tolerance design, economic models for correlated variables, burn-in, and group testing.

Pharmaceutical applications of RD and TD during granulation process, tablet pressing process, and coating process require a careful implementation because the primary characteristics of interest are often destructive. The primary purpose of this paper is to propose a new design modeling and optimization framework for the optimal factor setting and specification limits by incorporating the concept of surrogate variables with $\mathrm{RD}$ and TD. An overview of the proposed robust-tolerance design approach is presented in Figure 1.

\section{Development of a Robust Design Method for Destructive Characteristics}

2.1. Modification of Response Surface Methodology. Researchers have sought to combine Taguchi's robust design 
principles with conventional response surface methodology (RSM) to model the response directly as a function of design variables. a second-order polynomial model is adequate to accommodate the curvature of the process mean and variance functions, as evidenced by $[3,9,11,13,14,16-19]$. When the nature of a quality characteristic is destructive, a surrogate variable which is correlated to the quality characteristic is applied in many industrial applications. The response surface model associates control factors $x$ with the correlation between destructive variables $y$ and surrogate variables $t$. $y$ and $t$ are treated as random variables whose means depend upon the fixed values of $x$. Based on the dual response approach [9], the following models are recommended for destructive and surrogate variables:

$$
\begin{aligned}
& y(x)=\alpha_{0}+a_{1} x+a_{2} x^{2}+\varepsilon_{y}, \\
& t(x)=b_{0}+b_{1} x+b_{2} x^{2}+\varepsilon_{t},
\end{aligned}
$$

where $\varepsilon_{y}$ and $\varepsilon_{t}$ are normal random variables with mean zero and variances $\sigma_{y \varepsilon}^{2}$ and $\sigma_{t \varepsilon}^{2}$, respectively; and $\alpha_{0}, a_{1}$, $a_{2}, b_{0}, b_{1}$, and $b_{2}$ are model parameters. Based on the experimental results, the relationships between destructive quality characteristics and surrogate variables can be found as functional forms. Expanding the dual response approach, the estimated process mean functions of the performance variable $\widehat{\mu}_{y}$, and that of the surrogate variable $\widehat{\mu}_{t}$, the estimated variance function of the performance variable $\widehat{\sigma}_{y}^{2}$ and that of the surrogate variable $\widehat{\sigma}_{t}^{2}$, and the estimated correlation coefficient function between the performance variable and the surrogate variable $\hat{\rho}_{y t}$ are given as follows:

$$
\begin{gathered}
\widehat{\mu}_{y}(x)=\alpha_{y 0}+\alpha_{y 1} x+\alpha_{y 2} x^{2}, \\
\widehat{\mu}_{t}(x)=\alpha_{t 0}+\alpha_{t 1} x+\alpha_{t 2} x^{2}, \\
\widehat{\sigma}_{y}^{2}(x)=\beta_{y 0}+\beta_{y 1} x+\beta_{y 1} x^{2}, \\
\hat{\sigma}_{t}^{2}(x)=\beta_{t 0}+\beta_{t 1} x+\beta_{t 1} x^{2}, \\
\hat{\rho}_{y t}(x)=\gamma_{0}+\gamma_{1} x+\gamma_{2} x^{2},
\end{gathered}
$$

where $x$ is a design factor (or a control factor); $\alpha_{y 0}, \alpha_{y 1}$, and $\alpha_{y 2}$ are estimated regression coefficients of $\hat{\mu}_{y} ; \alpha_{t 0}, \alpha_{t 1}$, and $\alpha_{t 2}$ are estimated regression coefficients of $\widehat{\mu}_{t} ; \beta_{y 0}, \beta_{y 1}$, and $\beta_{y 2}$ are estimated regression coefficients of $\widehat{\sigma}_{y}^{2} ; \beta_{t 0}, \beta_{t 1}$, and $\beta_{t 2}$ are estimated regression coefficients of $\hat{\sigma}_{t}^{2}$; and $\gamma_{0}, \gamma_{1}$, and $\gamma_{2}$ are estimated regression coefficients of $\hat{\rho}_{y t}$. To determine the regression coefficients, ordinary methods, such as least square method, could be used. An experimental format for destructive quality characteristics and their associated surrogate variables is shown in Table $1 . \bar{y}$ and $s_{y}^{2}$ are the sample mean and the sample variance of destructive quality characteristic $y ; \bar{t}$ and $s_{t}^{2}$ are the sample mean and the sample variance of surrogate variable $t$; and $\rho_{y t}$ is the sample correlation coefficient associated with the destructive quality characteristic $y$ and the surrogate variable $t$.
2.2. Variability Reduction of Destructive Characteristics. From the experimental data, the functions of process mean $\widehat{\mu}(x)$ and variance $\widehat{\sigma}(x)$ are separately estimated by using the dual response approach. This model implies that the process mean is adjusted to the target $\tau_{y}$ first and then the variability is minimized. Because the primarily role of the $\mathrm{RD}$ approach in this paper is to reduce the variability of destructive quality characteristics, these destructive quality characteristics are considered in the $\mathrm{RD}$ stage, and their associated surrogate variables are considered in the TD stage for cost reduction. The RD optimization model associated with destructive quality characteristics $y$ is formulated as follows:

$\begin{array}{cc}\text { Minimize } & \widehat{\sigma}_{y}^{2}(\mathbf{x}) \\ \text { Subject to } & \mu_{y}(\mathbf{x})=\tau_{y} \\ & x \in \Omega,\end{array}$

where $\tau_{y}$ is a process target of destructive quality characteristic $y, \Omega$ is $\left\{x \in S: g_{i}(x) \leq 0, i=1,2, \ldots, n\right\}, S$ is a convex set in $R^{n}$, and $g_{i}(\mathbf{x})$ is the $i$ th constraint associated with the destructive quality characteristic.

\section{Development of an Economic Tolerance Design Method for Destructive Characteristics}

Functional performance and cost are two factors primarily affecting the design of tolerances [20]. Thus, the determination of optimal tolerance involves a tradeoff between the level of quality (based on functional performance) and costs (associated with the tolerance). In manufacturing processes, the following tolerances could be specified on quality characteristics:

(i) a tight tolerance which causes slow processing rates and high manufacturing costs due to additional manufacturing operations, additional care on part of operators, and need of expensive measuring and processing equipments,

(ii) a loose tolerance which causes low manufacturing costs while decreasing product/process quality.

In order to facilitate the economic tradeoff, researchers typically express quality in monetary terms using quality loss functions. As a result, proper implementation of the TD optimization under $100 \%$ inspections might lead to a substantial cost savings in scrap and rework costs and provide consistently high product quality throughout the manufacturing process. Ultimately, the selection of specification limits for a product is particularly important, since it directly affects the process defective rate, rejection cost, and loss to the customer due to the variability of product performance.

TD involves the problem of determining optimal specification limits from the viewpoint of cost reduction and functional performance. For example, Chase et al. [21], Kim and Cho [22], Speckhart [23], and Spotts [24] considered 
TABLE 1: Experimental format.

\begin{tabular}{|c|c|c|c|c|c|c|c|c|c|c|c|c|c|c|c|c|c|}
\hline \multirow{2}{*}{ Run No. } & \multicolumn{4}{|c|}{ Control factors $(x)$} & \multicolumn{6}{|c|}{ Quality characteristics $(y)$} & \multicolumn{6}{|c|}{ Surrogate variables $(t)$} & \multirow{2}{*}{$\rho_{y t}$} \\
\hline & $x_{1}$ & $x_{2}$ & $\cdots$ & $x_{p}$ & $y_{1}$ & $y_{2}$ & $\cdots$ & $y_{q}$ & $\mu_{y}$ & $s_{y}^{2}$ & $t_{1}$ & $t_{2}$ & $\cdots$ & $t_{q}$ & $\mu_{t}$ & $s_{t}^{2}$ & \\
\hline 1 & $x_{11}$ & $x_{21}$ & $\cdots$ & $x_{p 1}$ & $y_{11}$ & $y_{21}$ & $\cdots$ & $y_{q 1}$ & $\mu_{y 1}$ & $s_{y 1}^{2}$ & $t_{11}$ & $t_{21}$ & $\ldots$ & $t_{q 1}$ & $\mu_{t 1}$ & $s_{t 1}^{2}$ & $\rho_{y t 1}$ \\
\hline 2 & $x_{12}$ & $x_{22}$ & $\ldots$ & $x_{p 2}$ & $y_{12}$ & $y_{22}$ & $\ldots$ & $y_{q 2}$ & $\mu_{y 2}$ & $s_{y 2}^{2}$ & $t_{12}$ & $t_{22}$ & $\ldots$ & $t_{q 2}$ & $\mu_{t 2}$ & $s_{t 2}^{2}$ & $\rho_{y t 2}$ \\
\hline$\cdot$ & $\cdot$ & $\cdot$ & $\cdot$ & 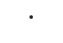 & 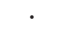 & · & $\cdot$ & · & $\cdot$ & $\cdot$ & . & $\cdot$ & $\cdot$ & $\cdot$ & $\cdot$ & $\cdot$ & $\cdot$ \\
\hline · & · & $\cdot$ & · & · & · & · & · & $\cdot$ & · & $\cdot$ & · & · & $\cdot$ & $\cdot$ & · & $\cdot$ & $\cdot$ \\
\hline$\cdot$ & $\cdot$ & . & $\cdot$ & $\cdot$ & . & . & . & $\cdot$ & . & . & . & . & $\cdot$ & $\cdot$ & . & $\cdot$ & . \\
\hline$n$ & $x_{1 n}$ & $x_{2 n}$ & $\cdots$ & $x_{p n}$ & $y_{1 n}$ & $y_{2 n}$ & $\cdots$ & $y_{q n}$ & $\mu_{y n}$ & $s_{y n}^{2}$ & $t_{1 n}$ & $t_{2 n}$ & $\cdots$ & $t_{q n}$ & $\mu_{t n}$ & $s_{t n}^{2}$ & $\rho_{y t n}$ \\
\hline
\end{tabular}

the reduction of manufacturing cost in tolerance allocation problems whereas Fathi [25], Kim and Cho [26], and Phillips and Cho [27] studied the issue of TD from the viewpoint of functional performance and expressing it in monetary terms by using Taguchi quality loss concept. In an integrated study considering the effect of both cost reduction and functional performance, Tang [28] developed an economic model for selecting the most profitable tolerance in situations where inspection cost is a linear function. When two viewpoints, such as cost and functional performance, are integrated, a tradeoff is often necessary. Along this line, Jeang [29] considered the simultaneous optimization of manufacturing cost, rejection cost, and quality loss by using a process capability index to establish a relationship between tolerance and standard deviation. When historical data of losses are available, a regression analysis could be applied, as exemplified by Phillips and Cho [27], who studied the minimization of the quality loss and the rejection cost. They developed optimization models using the first-order and second-order empirical loss functions. Wang and Liang [30] studied a tolerance optimization problem in the context of machining processes, such as milling, turning, drilling, reaming, boring, and grinding. Recently, Shin and Cho [31] studied two separate process parameters, such as process mean and variability, in the biobjective framework. Peng et al. [32] and Prabhaharan et al. [33] considered optimal process tolerances and stack-up conditions for mechanical assemblies as a combinatorial optimization problem. Destructive characteristics are often required in many situations, for instance, glass industry. Thus, an implementation of surrogate variables might be more practical. For surrogate variable-based TD optimization procedures, readers could refer to [17, 34-46].

3.1. Tolerance Design and Surrogate Variables. If the nature of major quality characteristics involves destructive testing, it is impossible to inspect all major quality characteristics themselves. In this case, it is more economical to use surrogate variables highly correlated with the major quality characteristics and relatively inexpensive to measure as screening variables. Let $h(y, t)$ denoting the joint distribution of major quality characteristic $y$ and surrogate variable $t$. Then, $h(y, t)$ assumed to be a bivariate normal distribution with means $\mu_{y}$ and $\mu_{t}$, variances $\sigma_{y}^{2}$ and $\sigma_{t}^{2}$, and correlation coefficient $\rho_{y t}(=$ $\left.\sigma_{y t} / \sigma_{y} \sigma_{t}\right)$. In the $\mathrm{RD}$ stage, the optimal setting of control factors is determined by setting all the major characteristics to their target values. Therefore, the values of these parameters $\mu_{y}, \mu_{t}, \sigma_{y}^{2}, \sigma_{t}^{2}$, and $\rho_{y t}$ are already known [47]. Note that $h(y, t)=g(y \mid t) f(t)$ where $f(t)$ is the marginal density function of $T$ and $g(y \mid t)$ is the conditional density function of $y$, given $T=t$. It can be shown that $f(t)$ is a normal density function with mean $\mu_{t}$ and variance $\sigma_{t}^{2}$, and $g(y \mid t)$ is a normal density function with mean $\mu=\mu_{y}+\rho_{y t}\left(\sigma_{y} / \sigma_{t}\right)\left(t-\mu_{t}\right)$ and variance $\sigma^{2}=\sigma_{y}^{2}\left(1-\rho_{y t}^{2}\right)$.

TD determines the tolerance limit of surrogate variables by minimizing cost incurred by both customer and manufacturer sides [47]. In the side of customers, it has been assumed that if the performance of a product falls within a tolerance limit, the product is acceptable to customers and its quality loss is zero. However, it is more reasonable to assume that quality loss occurs when a product performance deviates from its target although it is in the tolerance limit. Within the tolerance limit, the expected quality loss is evaluated by integrating a quality loss function and a probability density function over a region of interest. In this paper, the following quadratic quality loss function is applied:

$$
\text { Quality Loss }(y)=\theta(y-\tau)^{2} \text {, }
$$

where $\theta$ is a positive loss coefficient. Let LSL and USL be lower and upper bounds of a tolerance limit for surrogate variable $T$, respectively. By integrating the loss function defined in (4) and the product performance $h(y, t)$, the expected quality loss within a tolerance limit can be formulated as follows:

$$
\begin{aligned}
E[\text { Quality Loss }(y)] & =\int_{\text {LSL }}^{\text {USL }} \int_{-\infty}^{\infty} \theta(y-\tau)^{2} h(y, t) d y d t \\
& =\int_{\text {LSL }}^{\text {USL }} \theta\left(\sigma^{2}+(\mu-\tau)^{2}\right) f(t) d t
\end{aligned}
$$

From the definition of $g(y \mid t)$, after replacing $\mu$ with $\mu_{y}+$ $\rho_{y t}\left(\sigma_{y} / \sigma_{t}\right)\left(t-\mu_{t}\right)$ and $\sigma$ with $\sigma_{y}^{2}\left(1-\rho_{y t}^{2}\right),(5)$ becomes

$E[$ Quality Loss $(y)]$

$$
\begin{aligned}
=\theta \int_{\text {LSL }}^{\text {USL }}\left(\sigma_{y}^{2}\left(1-\rho_{y t}^{2}\right)\right. \\
\\
\left.+\left[\mu_{y}+\frac{\rho_{y t}^{2} \sigma_{y}\left(t-\mu_{t}\right)}{\sigma_{t}}-\tau\right]^{2}\right) f(t) d t .
\end{aligned}
$$


The rejection cost includes unit rework cost $C_{\text {rework }}$ and unit scrap cost $C_{\text {scrab }}$, which incur when a product performance falls above the upper specification limit and below the lower specification limit, respectively. The expected rework cost $E$ [Rework Cost] and the expected scrap cost $E[$ Scrap Cost $]$ are given as follows:

$$
\begin{aligned}
E[\text { Rework Cost }] & =C_{\text {rework }}(P[\mathrm{USL} \leq T]) \\
& =C_{\text {rework }} \int_{\mathrm{USL}}^{\infty} f(t) d t, \\
E[\text { Scrap Cost }] & =C_{\text {scrap }}(P[T \leq \mathrm{LSL}]) \\
& =C_{\text {scrap }} \int_{-\infty}^{\mathrm{LSL}} f(t) d t .
\end{aligned}
$$

To achieve tight tolerance, additional manufacturing operations and trainings, which probably decrease manufacturing processing rates and increase manufacturing cost, are usually required. The manufacturing cost contributes to a significant portion of the product unit cost, and its exclusion from the TD optimization model might result in a suboptimal tolerance. A tolerance range is defined in terms of $\delta, \mu_{t}$, and $\sigma_{t}$ as follows:

Tolerance Range $=$ USL - LSL

$$
=\left(\mu_{t}+\delta \sigma_{t}\right)-\left(\mu_{t}-\delta \sigma_{t}\right)=2 \delta \sigma_{t} .
$$

For simplicity, the manufacturing cost is assumed to have a linear relationship with the tolerance range as described below:

$$
\text { Mfg Cost }=a_{0}+a_{1} \text { Tolerance Range }+\varepsilon_{\mathrm{mfg}},
$$

where $\varepsilon_{\mathrm{mfg}}$ represents the least-squares regression error. This linear manufacturing cost-tolerance model is also implemented in $[48,49]$. The expected manufacturing cost could then be written as:

$$
E[\text { Mfg Cost }]=a_{0}+a_{1} \text { Tolerance Range. }
$$

Substituting Tolerance Range $=2 \delta \sigma_{t}$ in (10), the expected manufacturing cost becomes

$$
E[\mathrm{Mfg} \text { Cost }]=a_{0}+2 a_{1} \delta \sigma_{t} .
$$

3.2. The Proposed Optimization Model for Tolerance Design. The objective of economic TD is to find the tolerance limit which minimizes the expected total cost including expected quality loss, expected rework cost, expected scrap cost, and expected manufacturing cost. Thus, the expected total cost is mathematically expressed as

$$
\begin{aligned}
E[\text { Total Cost }]= & E[\text { Quality Loss }(y)] \\
& +E[\text { Rework Cost }]+E[\text { Scrap Cost }] \\
& +E[\text { Mfg Cost }] .
\end{aligned}
$$

By replacing (6), (7), and (11) into (12), the expected total cost is reformulated as:

$$
\begin{aligned}
& E \text { [Total Cost] } \\
& =\theta \int_{\text {LSL }}^{\mathrm{USL}}\left(\sigma_{y}^{2}\left(1-\rho_{y t}^{2}\right)\right. \\
& \left.+\left[\mu_{y}+\frac{\rho_{y t}^{2} \sigma_{y}\left(t-\mu_{t}\right)}{\sigma_{t}}-\tau\right]^{2}\right) f(t) d t \\
& +C_{\text {rework }} \int_{\text {USL }}^{\infty} f(t) d t \\
& +C_{\text {scrap }} \int_{-\infty}^{\text {LSL }} f(t) d t \\
& +a_{0}+2 a_{1} \delta \sigma_{t} .
\end{aligned}
$$

To simplify (13), the marginal density function of $t$ is converted from normal distribution function $f(t)$ to standard normal distribution $\phi(z)$ by using

$$
z=\frac{\left(t-\mu_{t}\right)}{\sigma_{t}} .
$$

Thus, (13) could be rewritten as

$$
\begin{aligned}
E[\text { Total Cost }]= & \theta \int_{-\delta}^{\delta}\left(\sigma_{y}^{2}\left(1-\rho_{y t}^{2}\right)\right. \\
& \left.+\left(\mu_{y}+\rho_{y t} \sigma_{y} z-\tau\right)^{2}\right) \phi(z) d z \\
& +C_{\text {rework }} \int_{\delta}^{\infty} \phi(z) d z \\
& +C_{\text {scrap }} \int_{-\infty}^{-\delta} \phi(z) d z+a_{0}+2 a_{1} \sigma_{t} \delta,
\end{aligned}
$$

where USL $=\mu_{y}+\delta \sigma_{y}$, LSL $=\mu_{y}-\delta \sigma_{y}$, and $\delta>0$. Based on the dual response approach used in the $\mathrm{RD}$ stage, terms $\mu_{y}$ and $\tau$ are removed from (15). Also, after applying the following properties of normal probability density functions:

$$
\begin{aligned}
& \int_{-\infty}^{r} \phi(z) d z=\Phi(r), \quad \int_{-\infty}^{r} z \phi(z) d z=-\phi(r), \\
& \int_{-\infty}^{r} z^{2} \phi(z) d z=\Phi(r)-r \phi(r),
\end{aligned}
$$

the expected total cost becomes

$$
\begin{aligned}
E[\text { Total Cost }]= & 2 a_{1} \sigma_{t} \delta-2 \rho_{y t}^{2} \sigma_{y}^{2} \theta \delta \phi(\delta) \\
& +\left\{2 \sigma_{y}^{2}\left(1-\rho_{y t}^{2}\right) \theta+2 \rho_{y t}^{2} \sigma_{y}^{2} \theta\right. \\
& \left.-C_{\text {rework }}-C_{\text {scrap }}\right\} \Phi(\delta) \\
& +C_{\text {rework }}+C_{\text {scrap }}-\theta \sigma_{y}^{2}+a_{0} .
\end{aligned}
$$


3.3. Determination of the Closed-Form Solution for Tolerance Optimization. To investigate the optimal solution of tolerance design, the first derivative with respect to $\delta$ is as follows:

$$
\begin{aligned}
\frac{\partial E[\text { Total Cost }]}{\partial \delta}= & 2 a_{1} \sigma_{t}+\phi(\delta) \\
& \times\left[2 \sigma_{y}^{2}\left(1-\rho_{y t}^{2}\right) \theta-C_{\text {rework }}-C_{\text {scrap }}\right] \\
& +2 \rho_{y t}^{2} \sigma_{y}^{2} \theta \delta^{2} \phi(\delta) .
\end{aligned}
$$

Equating $\partial E[$ Total Cost $] / \partial \delta$ to zero and substituting $\phi(\delta)$ with $e^{(-1 / 2) \delta^{2}} / \sqrt{2 \pi}$, (18) becomes

$$
\begin{aligned}
2 a_{1} \sigma_{t} & +\left(2 \sigma_{y}^{2}\left(1-\rho_{y t}^{2}\right) \theta-C_{\text {rework }}-C_{\text {scrap }}+2 \rho_{y t}^{2} \sigma_{y}^{2} \theta \delta^{2}\right) \\
& \times\left(\frac{e^{-\delta^{2} / 2}}{\sqrt{2 \pi}}\right)=0 .
\end{aligned}
$$

Since it is nearly impossible to obtain a closed-form solution from this complex equation, Lambert $W$ function is introduced to effectively obtain such a solution for $\delta$ as discussed in Appendix B. According to the property of Lambert $W$ function $([20,50])$ such that

$$
\xi_{1}\left(\delta^{2}+\xi_{2}\right) e^{\xi_{3} \delta^{2}}=\xi_{4},
$$

where $\xi_{1}, \xi_{2}, \xi_{3}$, and $\xi_{4}$ are not functions of $\delta$, the optimal tolerance $\delta^{*}$ can be derived from the following relationship:

$$
\delta= \pm \sqrt{\frac{\text { Lambert } W\left(\xi_{3} \xi_{4} e^{\xi_{2} \xi_{3}} / \xi_{1}\right)}{\xi_{3}}-\xi_{2}}
$$

According to (20), (19) after formatting becomes

$$
\begin{aligned}
\left(\delta^{2}\right. & \left.+\frac{2 \sigma_{y}^{2}\left(1-\rho_{y t}^{2}\right) \theta-C_{\text {rework }}-C_{\text {scrap }}}{2 \rho_{y t}^{2} \sigma_{y}^{2} \theta}\right) e^{-\delta^{2} / 2} \\
& =\frac{-a_{1} \sigma_{t} \sqrt{2 \pi}}{\rho_{y t}^{2} \sigma_{y}^{2} \theta} .
\end{aligned}
$$

$\xi_{1}, \xi_{2}, \xi_{3}$, and $\xi_{4}$ in (21) can be replaced with $1,\left[2 \sigma_{y}^{2}(1-\right.$ $\left.\left.\rho_{y t}^{2}\right) \theta-C_{\text {rework }}-C_{\text {scrap }}\right] / 2 \rho_{y t}^{2} \sigma_{y}^{2} \theta,-1 / 2$, and $-a_{1} \sigma_{t} \sqrt{2 \pi} / \rho_{y t}^{2} \sigma_{y}^{2} \theta$, respectively. Then, the closed-form solution of $\delta^{*}$ is formulated and provided as follows:

$$
\begin{aligned}
\delta^{*}= \pm( & -2 \text { Lambert } W \\
& \left(\frac{a_{1} \sigma_{1} \sqrt{2 \pi} e^{\left(\left(C_{\text {rework }}+C_{\text {scrap }}-2 \sigma_{y}^{2}\left(1-\rho_{y t}^{2}\right) \theta\right) /\left(4 \rho_{y t}^{2} \sigma_{y}^{2} \theta\right)\right)}}{2 \rho_{y t}^{2} \sigma_{y}^{2} \theta}\right) \\
& \left.+\left(\frac{C_{\text {rework }}+C_{\text {scrap }}-2 \sigma_{y}^{2}\left(1-\rho_{y t}^{2}\right) \theta}{2 \rho_{y t}^{2} \sigma_{y}^{2} \theta}\right)\right)^{1 / 2},
\end{aligned}
$$

where $\sigma_{y}^{2}, \sigma_{t}^{2}, \rho_{y t}, \theta, C_{\text {rework }}$ and $C_{\text {scrap }}$; are the optimal variance of the destructive quality characteristic $y$, the optimal variance of the surrogate variable $t$, the correlation coefficient associated with the destructive quality characteristic $y$ and the surrogate variable $t$, the positive loss coefficient, unit rework cost, and unit scrap cost, respectively. Although the closed-form solution shown in (23) consists of the Lambert $W$ function, its computation is remarkably simple. After $\delta^{*}$ is obtained, the optimal LSL and USL can be derived from $\mu_{t}^{*}-\delta^{*} \sigma_{t}^{*}$ and $\mu_{t}^{*}+\delta^{*} \sigma_{t}^{*}$, respectively.

3.4. Determination of the Conditions for Convexity. To verify the validity of the optimal solution, the second derivative is computed and the conditions for obtaining the minimum value of $E$ [Total Cost] are investigated. The second derivative of $E$ [Total Cost] with respect to $\delta$ is as follows:

$$
\begin{aligned}
\frac{\partial^{2} E[\text { Total Cost }]}{\partial \delta^{2}}= & \delta \phi(\delta) \\
& \times\left[C_{\text {rework }}+C_{\text {scrap }}-2 \sigma_{y}^{2} \theta\right. \\
& \left.\quad \times\left(1-3 \rho_{y t}^{2}+\rho_{y t}^{2} \delta^{2}\right)\right] .
\end{aligned}
$$

The value of $E$ [Total Cost] at the stationary points will be minimum if the second derivative is greater than or equal to zero. Therefore, the interval for a minimum value of $\delta$ could be written as

$$
0 \leq \delta \leq \sqrt{\frac{C_{\text {rework }}+C_{\text {scrap }}-2 \sigma_{y}^{2} \theta}{2 \rho_{y t}^{2} \sigma_{y}^{2} \theta}+3 .}
$$

In order to avoid imaginary values, the following condition needs to be satisfied:

$$
\frac{C_{\text {rework }}+C_{\text {scrap }}-2 \sigma_{y}^{2} \theta}{2 \rho_{y t}^{2} \sigma_{y}^{2} \theta} \geq-3
$$

If both conditions in (25) and (26) are satisfied, the closedform solution of $\delta^{*}$ defined in (23) represents the global minimum of $E$ [Total Cost]. The overall framework of TD optimization and its associated key elements are provided as follows.

\section{Given Parameters}

$f(t)$ : the normal probability density function of surrogate variable $t$,

$\tau$ : the target of the process mean of destructive quality characteristic $y$, 
$\mu_{\dot{y}}$ the optimal process mean of destructive quality characteristic $y$, derived from the RD stage,

$\sigma_{y}^{2}$ : the optimal variance of destructive quality characteristic $y$, derived from the $\mathrm{RD}$ stage,

$\mu_{t}$ : the optimal process mean of surrogate variable $t$, derived from the RD stage,

$\sigma_{t}^{2}$ : the optimal variance of surrogate variable $t$, derived from the RD stage,

$\rho_{y t}$ : the optimal correlation coefficient between destructive quality characteristic $y$ and surrogate variable $t$, derived from the RD stage.

\section{System Parameters}

$$
\begin{aligned}
E[\text { Total Cost }]= & E[\text { Quality Loss }] \\
& +E[\text { Mfg Cost }] \\
& +E[\text { Rework Cost }] \\
& +E[\text { Scrap Cost }]
\end{aligned}
$$

$E[$ Quality Loss $(y)]$

$$
\begin{aligned}
& =\theta \int_{\text {LSL }}^{\mathrm{USL}}\left(\sigma_{y}^{2}\left(1-\rho_{y t}^{2}\right)\right. \\
& +\left\{\mu_{y}\right. \\
& \left.\left.+\frac{\rho_{y t}^{2} \sigma_{y}\left(t-\mu_{t}\right)}{\sigma_{t}}-\tau\right\}^{2}\right) f(t) d t, \\
& E[\text { Mfg Cost }]=a_{0}+2 a_{1} \delta \sigma_{t}, \\
& E[\text { Rework Cost }]=C_{\text {rework }} \int_{\mathrm{USL}}^{\infty} f(t) d t, \\
& E[\text { Scrap Cost }]=C_{\text {scrap }} \int_{-\infty}^{\mathrm{LSL}} f(t) d t .
\end{aligned}
$$

Find

$\delta^{*}:$ the optimal tolerance, which leads to the derivation of USL and LSL as follows:

$$
\mathrm{USL}=\mu_{t}+\delta^{*} \sigma_{t}, \quad \mathrm{LSL}=\mu_{t}-\delta^{*} \sigma_{t} .
$$

Minimize

$$
\begin{aligned}
E[\text { Total Cost }]= & 2 a_{1} \sigma_{t} \delta-2 \rho_{y t}^{2} \sigma_{y}^{2} \theta \delta \phi(\delta) \\
& +\left\{2 \sigma_{y}^{2}\left(1-\rho_{y t}^{2}\right) \theta+2 \rho_{y t}^{2} \sigma_{y}^{2} \theta\right. \\
& \left.\quad-C_{\text {rework }}-C_{\text {scrap }}\right\} \Phi(\delta) \\
& +C_{\text {rework }}+C_{\text {scrap }}-\theta \sigma_{y}^{2}+a_{0} .
\end{aligned}
$$

Closed-Form Solution

$$
\begin{aligned}
& \delta^{*}= \pm(- \text { Lambert } W \\
& \times\left(\frac{a_{1} \sigma_{1} \sqrt{2 \pi} e^{\left(\left(C_{\text {rework }}+C_{\text {scrap }}-2 \sigma_{y}^{2}\left(1-\rho_{y t}^{2}\right) \theta\right) / 4 \rho_{y t}^{2} \sigma_{y}^{2} \theta\right)}}{2 \rho_{y t}^{2} \sigma_{y}^{2} \theta}\right) \\
&\left.+\left(\frac{C_{\text {rework }}+C_{\text {scrap }}-2 \sigma_{y}^{2}\left(1-\rho_{y t}^{2}\right) \theta}{2 \rho_{y t}^{2} \sigma_{y}^{2} \theta}\right)\right)^{1 / 2}
\end{aligned}
$$

\section{Numerical Example}

To illustrate the application of the proposed approach, a problem of measuring tablet hardness in pharmaceutical manufacturing process is provided. Three factors, which are pressure $\left(x_{1}, \mathrm{MPa}\right)$, humidity $\left(x_{2}, \%\right)$, and compression speed $\left(x_{3}, \mathrm{~mm} / \mathrm{min}\right)$, were in control. The hardness $(y, \mathrm{~kg})$, required at five $[18,51]$, was considered as a destructive quality characteristic because the tablet could be easily destroyed during the measurement. Process Analytical Technology (PAT) tools such as Raman, Near-Infrared (NIR), and Focused Beam Reflectance Measurement (FBRM) were used to understand and control the pharmaceutical process. In addition, Terahertz Pulsed Spectroscopy (TPS) and Terahertz Pulsed Imaging (TPI) techniques were used and terahertz refractive index $(t)$ was introduced as a surrogate variable for the tablet hardness $(y)[18,51]$.

From the overview of the proposed robust-tolerance approach, provided in Figure 1, there are three main steps: process measurement, RD optimization, and TD optimization with Lambert $W$ function. Central composite design (CCD) with three replications was applied to collect the experimental data of the destructive quality characteristic $y$ and its associated surrogate variable $t$. Then, the sample mean of $y\left(\mu_{y}\right)$, the sample variance of $y\left(s_{y}^{2}\right)$, the sample mean of $t\left(\mu_{t}\right)$, the sample variance of $t\left(s_{t}^{2}\right)$, and the correlation coefficient of $y$ and $t\left(\rho_{y t}\right)$ were calculated. Table 2 shows all the experimental data obtained from step one. The other two steps are explained in Sections 4.1 and 4.2, respectively.

4.1. Stage I: Robust Design Optimization. By applying RSM to the experimental data shown in Table 2, fitted functions of the mean and the variance of $y$, the mean and the variance of 
TABLE 2: Experimental data for a pharmaceutical manufacturing process.

\begin{tabular}{|c|c|c|c|c|c|c|c|c|c|c|c|c|c|}
\hline \multicolumn{3}{|c|}{ Control factors $(x)$} & \multicolumn{5}{|c|}{ Destructive quality characteristic hardness $(y)$} & \multicolumn{5}{|c|}{ Surrogate variable terahertz refractive index $(t)$} & \multirow{2}{*}{$\rho_{y t}$} \\
\hline$x_{1}$ & $x_{2}$ & $x_{3}$ & $y_{1}$ & $y_{2}$ & $y_{3}$ & $\mu_{y}$ & $s_{y}^{2}$ & $t_{1}$ & $t_{2}$ & $t_{3}$ & $\mu_{t}$ & $s_{t}^{2}$ & \\
\hline-1 & -1 & -1 & 6.478 & 7.518 & 6.281 & 6.759 & 0.442 & 1.477 & 1.560 & 1.407 & 1.481 & 0.006 & 0.948 \\
\hline-1 & -1 & 1 & 2.000 & 3.004 & 2.345 & 2.450 & 0.260 & 1.043 & 1.180 & 1.000 & 1.074 & 0.009 & 0.840 \\
\hline-1 & 1 & -1 & 8.839 & 10.220 & 9.088 & 9.382 & 0.542 & 1.670 & 1.787 & 1.641 & 1.699 & 0.006 & 0.937 \\
\hline-1 & 1 & 1 & 5.699 & 6.750 & 5.581 & 6.010 & 0.414 & 1.403 & 1.460 & 1.284 & 1.382 & 0.008 & 0.807 \\
\hline 1 & -1 & -1 & 5.495 & 6.817 & 6.280 & 6.197 & 0.443 & 1.351 & 1.505 & 1.343 & 1.400 & 0.008 & 0.779 \\
\hline 1 & -1 & 1 & 9.600 & 10.570 & 9.103 & 9.757 & 0.554 & 1.753 & 1.801 & 1.648 & 1.734 & 0.006 & 0.922 \\
\hline 1 & 1 & -1 & 4.987 & 5.985 & 4.807 & 5.260 & 0.403 & 1.327 & 1.362 & 1.205 & 1.298 & 0.007 & 0.774 \\
\hline 1 & 1 & 1 & 10.756 & 11.940 & 10.510 & 11.070 & 0.586 & 1.903 & 2.000 & 1.866 & 1.923 & 0.005 & 0.994 \\
\hline-1.68 & 0 & 0 & 7.113 & 7.848 & 6.443 & 7.135 & 0.494 & 1.480 & 1.523 & 1.380 & 1.461 & 0.005 & 0.968 \\
\hline 1.68 & 0 & 0 & 7.473 & 8.470 & 7.143 & 7.695 & 0.477 & 1.570 & 1.626 & 1.466 & 1.554 & 0.007 & 0.900 \\
\hline 0 & -1.68 & 0 & 8.657 & 9.609 & 8.195 & 8.820 & 0.520 & 1.655 & 1.718 & 1.567 & 1.647 & 0.006 & 0.957 \\
\hline 0 & 1.68 & 0 & 10.647 & 12.000 & 10.557 & 11.070 & 0.653 & 1.828 & 1.936 & 1.804 & 1.856 & 0.005 & 0.993 \\
\hline 0 & 0 & -1.68 & 2.705 & 3.804 & 3.089 & 3.199 & 0.312 & 1.082 & 1.213 & 1.050 & 1.115 & 0.007 & 0.858 \\
\hline 0 & 0 & 1.68 & 7.779 & 8.888 & 7.546 & 8.071 & 0.514 & 1.610 & 1.657 & 1.490 & 1.586 & 0.007 & 0.820 \\
\hline 0 & 0 & 0 & 8.431 & 8.511 & 7.271 & 8.071 & 0.482 & 1.651 & 1.561 & 1.475 & 1.563 & 0.008 & 0.828 \\
\hline 0 & 0 & 0 & 7.379 & 7.286 & 6.173 & 6.946 & 0.451 & 1.475 & 1.551 & 1.383 & 1.470 & 0.007 & 0.858 \\
\hline 0 & 0 & 0 & 6.993 & 7.887 & 6.521 & 7.134 & 0.481 & 1.519 & 1.549 & 1.392 & 1.487 & 0.007 & 0.868 \\
\hline 0 & 0 & 0 & 8.856 & 8.856 & 7.623 & 8.445 & 0.507 & 1.700 & 1.609 & 1.527 & 1.612 & 0.008 & 0.849 \\
\hline 0 & 0 & 0 & 7.299 & 8.657 & 7.694 & 7.883 & 0.488 & 1.537 & 1.654 & 1.480 & 1.557 & 0.008 & 0.818 \\
\hline 0 & 0 & 0 & 10.443 & 10.300 & 9.092 & 9.945 & 0.550 & 1.739 & 1.788 & 1.631 & 1.720 & 0.006 & 0.918 \\
\hline
\end{tabular}

$t$, and the correlation coefficient of $y$ and, $t$ are obtained and respectively, provided as follows:

$$
\begin{aligned}
\widehat{\mu}_{y}= & 8.09+0.631 x_{1}+0.757 x_{2}+0.724 x_{3} \\
& -0.726 x_{1} x_{2}+2.13 x_{1} x_{3}+0.398 x_{2} x_{3} \\
& -0.344 x_{1}^{2}+0.551 x_{2}^{2}-0.973 x_{3}^{2} \\
\widehat{\sigma}_{y}^{2}= & 0.494+0.0219 x_{1}+0.0344 x_{2}+0.0237 x_{3} \\
& -0.0328 x_{1} x_{2}+0.0755 x_{1} x_{3}+0.0157 x_{2} x_{3} \\
& -0.0111 x_{1}^{2}+0.0246 x_{2}^{2}-0.0367 x_{3}^{2} ; \\
\widehat{\mu}_{t}= & 1.57+0.0641 x_{1}+0.0706 x_{2}+0.0752 x_{3} \\
& -0.0549 x_{1} x_{2}+0.210 x_{1} x_{3}+0.0476 x_{2} x_{3} \\
& -0.0290 x_{1}^{2}+0.0573 x_{2}^{2}-0.0845 x_{3}^{2} ; \\
\widehat{\sigma}_{t}^{2}= & 0.00731+0.000027 x_{1}-0.000343 x_{2}+0.000073 x_{3} \\
& -0.000125 x_{1} x_{2}-0.00112 x_{1} x_{3}-0.000125 x_{2} x_{3} \\
& -0.000307 x_{1}^{2}-0.000484 x_{2}^{2}+0.000046 x_{3}^{2}, \\
\widehat{\rho}_{y t}= & 0.858-0.0130 x_{1}+0.0061 x_{2}+0.0045 x_{3} \\
& +0.0139 x_{1} x_{2}+0.0751 x_{1} x_{3}+0.0069 x_{2} x_{3} \\
& +0.0180 x_{1}^{2}+0.0325 x_{2}^{2}-0.0156 x_{3}^{2} .
\end{aligned}
$$

To demonstrate the statistical analysis, ANOVA for the five responses based on significant level $\alpha$ at 0.05 was conducted as shown in Tables 3, 4, 5, 6, and 7. The result could be summarized as follows. For $\widehat{\mu}_{y}$, two main effects (i.e. $x_{2}$ and $x_{3}$ ), the interaction between $x_{1}$ and $x_{3}$, and the quadratic effect of $x_{3}$ are significant; for $\widehat{\sigma}_{y}^{2}, x_{2}$, the interaction between $x_{1}$ and $x_{3}$, and the quadratic effect of $x_{3}$ are significant; for $\widehat{\mu}_{t}$, all the main effects (i.e. $x_{1}, x_{2}$, and $x_{3}$ ), the interaction between $x_{1}$ and $x_{3}$, and the quadratic effect of $x_{3}$ are significant; for $\widehat{\sigma}_{t}^{2}$, only the interaction between $x_{1}$ and $x_{3}$ is significant; and for $\hat{\rho}_{y t}$, the interaction between $x_{1}$ and $x_{3}$ and the quadratic effect of $x_{2}$ are significant.

The variability of destructive quality characteristic $y$ could be reduced by applying the $\mathrm{RD}$ model shown in (3) with $\tau_{y}=5$. The optimal solutions (i.e. $x_{1}^{*}=-0.3000, x_{2}^{*}=$ -0.6219 , and $\left.x_{3}^{*}=-1.6810\right)$ and then the optimal parameters associated with $y$ and $t$ (i.e. $\mu_{y}^{*}=5.0000, \sigma_{y}^{2 *}=0.3792, \mu_{t}^{*}=$ 1.3061, $\sigma_{t}^{2 *}=0.0070$, and $\left.\rho_{y t}^{*}=0.8684\right)$ were obtained and provided in Table 8 . Figures 2, 3, and 4 present the graphical representations of all the fitted response functions (i.e. $\widehat{\mu}_{y}, \widehat{\sigma}_{y}^{2}$, $\widehat{\mu}_{t}, \widehat{\sigma}_{t}^{2}$, and $\hat{\rho}_{y t}$ ) with respect to the optimal setting of each input factor (i.e. $x_{1}^{*}, x_{2}^{*}$, and $x_{3}^{*}$, resp.).

4.2. Stage II: Tolerance Design Optimization. After the variability was reduced in the RD stage and the optimal setting for the hardness $(y)$ and the Terahertz Refractive Index $(t)$ were obtained, the proposed economic TD framework was 
TABLE 3: ANOVA of the mean of $y\left(\widehat{\mu}_{y}\right)$.

\begin{tabular}{lcccc}
\hline Predictor & Coef & SE Coef & $T$ & $P$ \\
\hline Constant & 8.0877 & 0.4857 & 16.65 & 0.000 \\
$x 1$ & 0.6315 & 0.3222 & 1.96 & 0.078 \\
$x 2$ & 0.7573 & 0.3222 & 2.35 & 0.041 \\
$x 3$ & 0.7236 & 0.3222 & 2.25 & 0.049 \\
$x 1 \times 2$ & -0.7259 & 0.4210 & -1.72 & 0.115 \\
$x 1 \times 3$ & 2.1314 & 0.4210 & 5.06 & 0.000 \\
$x 2 x 3$ & 0.3984 & 0.4210 & 0.95 & 0.366 \\
$x 1 x 1$ & -0.3435 & 0.3136 & -1.10 & 0.299 \\
$x 2 x 2$ & 0.5507 & 0.3136 & 1.76 & 0.110 \\
$x 3 x 3$ & -0.9727 & 0.3136 & -3.10 & 0.011 \\
\hline$S=1.1908$ & & & & $R^{2}($ adj $)=72.4 \%$ \\
\hline Source & & Analysis of variance & & \\
\hline Regression & DF & SS & MS & \\
Residual error & 9 & 83.371 & 9.263 & 0.53 \\
\hline Total & 10 & 14.18 & 1.418 & 0.004 \\
\hline
\end{tabular}

TABLE 4: ANOVA of the variance of $y\left(\widehat{\sigma}_{y}^{2}\right)$.

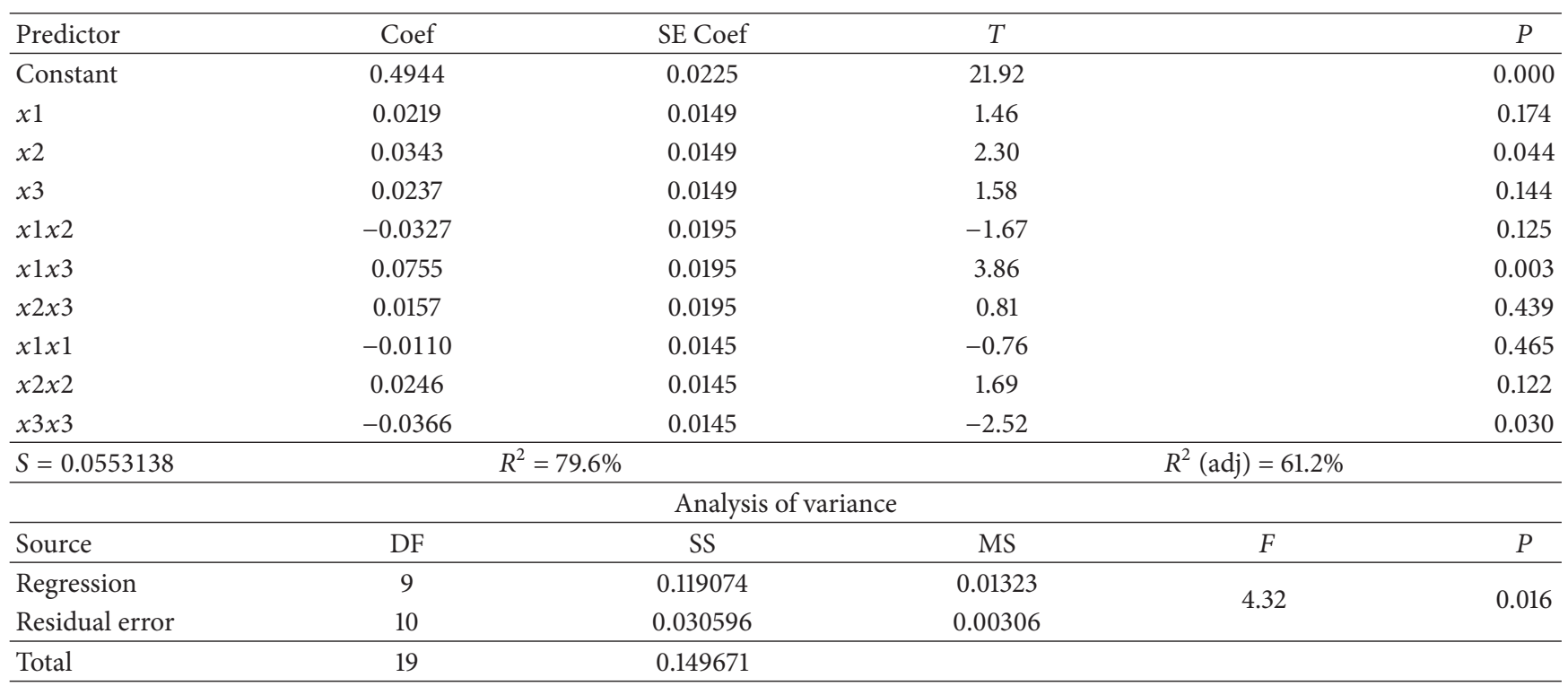

conducted with respect to $t$ in order to find the optimal tolerance $\delta^{*}$, which could be easily found by the closed-form solution shown in (23) and then lead to the derivation of the optimal lower and upper specification limits (i.e., LSL ${ }^{*}$ and $\left.\mathrm{USL}^{*}\right)$, respectively. When the inspection was implemented, the expected quality loss (E[Quality Loss $(y)])$ occurred as shown in (6) where the positive loss coefficient $\theta$ was 250. The expected manufacturing cost (E[Mfg Cost $])$ presented in (11) was described as a polynomial model where $a_{0}$ and $a_{1}$ were 100 and -0.2 , respectively. Also, when the product performance fell above the upper limit or below the lower limit of the production specification, the expected rework cost (E[Rework Cost]) and the expected scrap cost (E[Scrap Cost]) were calculated as, respectively, defined in (7) where both $C_{\text {rework }}$ and $C_{\text {scrap }}$ were set to an arbitrary value such as 80 . All the parameters, objectives, and optimal solutions involved in this TD stage are provided as follows.

Given Conditions

$\begin{array}{ll}f(t) & e^{-\left(\left(t-\mu_{t}\right)^{2} / 2 \sigma_{t}^{2}\right)} / \sqrt{2 \pi \sigma_{t}^{2}} \\ \tau & 5.0000 \\ \mu_{y} & 5.0000 \\ \sigma_{y}^{2} & 0.3792 \\ \mu_{t} & 1.3061 \\ \sigma_{t}^{2} & 0.0070 \\ \rho_{y t} & 0.8684 .\end{array}$


TABLe 5: ANOVA of the mean of $t\left(\widehat{\mu}_{t}\right)$.

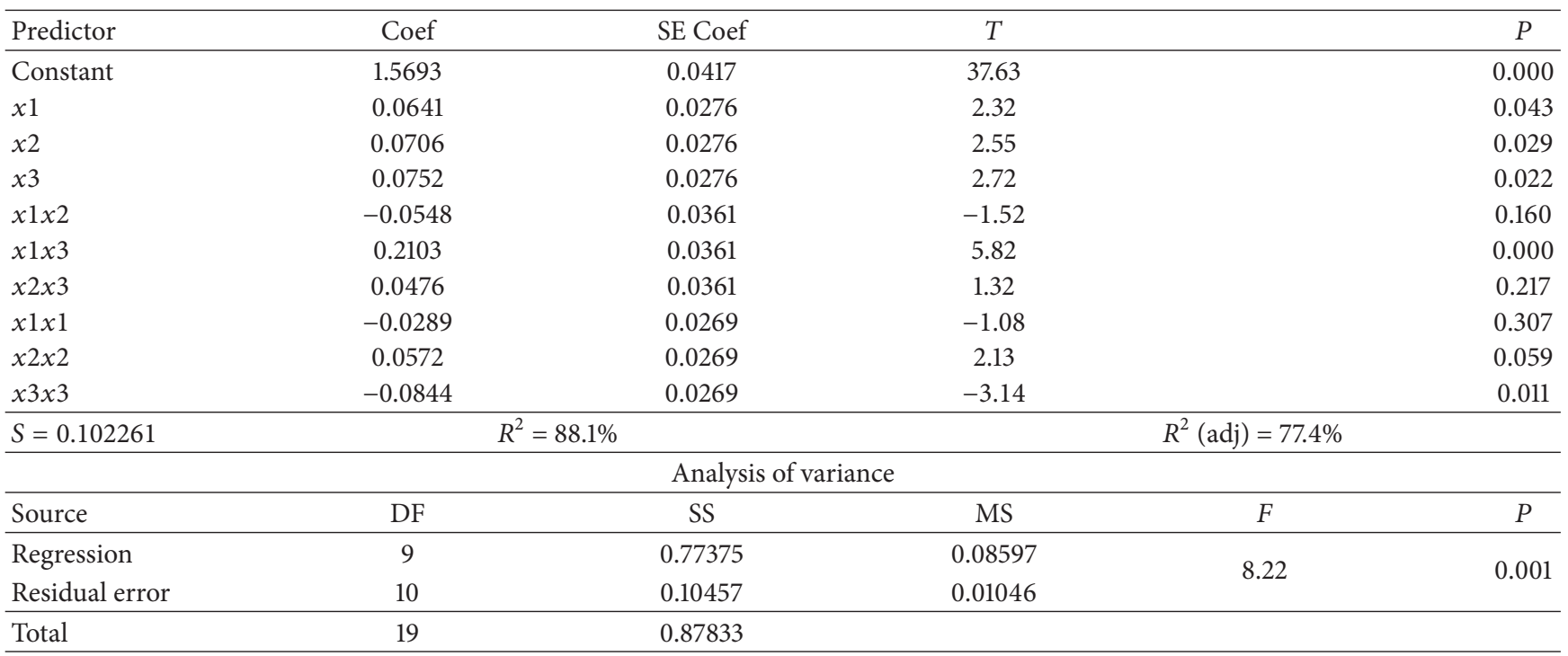

TABLE 6: ANOVA of the variance of $t\left(\widehat{\sigma}_{t}^{2}\right)$.

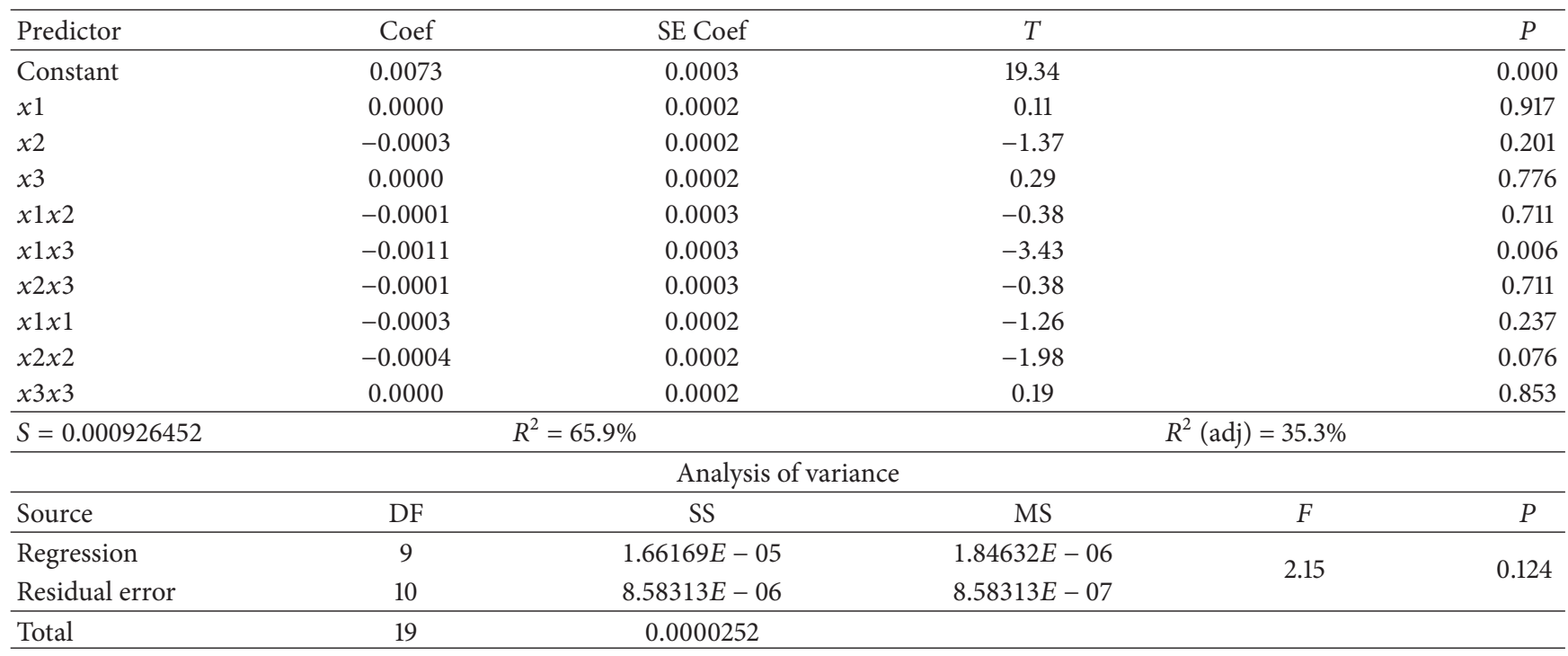

\section{System Parameters}

$$
\begin{aligned}
& E \text { [Total Cost }] \\
& =E[\text { Quality Loss }]+E[\text { Mfg Cost }] \\
& +E[\text { Rework Cost }] \\
& +E[\text { Scrap Cost }],
\end{aligned}
$$

$E[$ Quality Loss $(y)]$

$$
=250
$$

$$
\begin{aligned}
\times \int_{\text {LSL }}^{\mathrm{USL}}\left(\sigma_{y}^{2}\left(1-\rho_{y t}^{2}\right)\right. & \\
& \left.+\left\{\mu_{y}+\frac{\rho_{y t}^{2} \sigma_{y}\left(t-\mu_{t}\right)}{\sigma_{t}}-5\right\}^{2}\right)
\end{aligned}
$$

$$
\times f(t) d t
$$

$$
\begin{aligned}
E[\text { Mfg Cost }] & =100-0.4 \delta \sigma_{t} . \\
E[\text { Rework Cost }] & =80 \int_{\mathrm{USL}}^{\infty} f(t) d t, \\
E[\text { Scrap Cost }] & =80 \int_{-\infty}^{\mathrm{LSL}} f(t) d t,
\end{aligned}
$$

Find

$\delta^{*}$ : the optimal tolerance, which leads to the derivation of USL and LSL as follows:

$\mathrm{USL}=\mu_{t}+\delta^{*} \sigma_{t}, \quad \mathrm{LSL}=\mu_{t}-\delta^{*} \sigma_{t}$. 
TABLE 7: ANOVA of the correlation coefficient of $y$ and $t\left(\widehat{\rho}_{y t}\right)$.

\begin{tabular}{lcccc}
\hline Predictor & Coef & SE Coef & $T$ & $P$ \\
\hline Constant & 0.8579 & 0.0167 & 51.14 & 0.000 \\
$x 1$ & -0.0129 & 0.0111 & -1.17 & 0.270 \\
$x 2$ & 0.0061 & 0.0111 & 0.55 & 0.595 \\
$x 3$ & 0.0044 & 0.0111 & 0.40 & 0.696 \\
$x 1 \times 2$ & 0.0138 & 0.0145 & 0.95 & 0.363 \\
$x 1 \times 3$ & 0.0751 & 0.0145 & 5.17 & 0.000 \\
$x 2 x 3$ & 0.0068 & 0.0145 & 0.47 & 0.647 \\
$x 1 x 1$ & 0.0180 & 0.0108 & 1.66 & 0.127 \\
$x 2 x 2$ & 0.0325 & 0.0108 & 3.00 & 0.013 \\
$x 3 x 3$ & -0.0155 & 0.0108 & -1.44 & 0.181 \\
\hline$S=0.0411335$ & & & & $R^{2}($ adj $)=64.8 \%$ \\
\hline Source & & Analysis of variance & & \\
\hline Regression & DF & SS & MS & \\
Residual error & 9 & 0.074522 & 0.00828 & 0.001692 \\
\hline Total & 10 & 0.01692 & & \\
\hline
\end{tabular}

TABLE 8: Optimal solutions from the RD stage.

\begin{tabular}{ccccccr}
\hline & Control factors $(x)$ & \multicolumn{2}{c}{ Hardness $(y)$} & \multicolumn{3}{c}{ Terahertz refractive index $(t)$} \\
$x_{1}^{*}$ & $x_{2}^{*}$ & $x_{3}^{*}$ & $\mu_{y}^{*}$ & $\sigma_{y}^{2 *}$ & $\mu_{t}^{*}$ & $\sigma_{t}^{2 *}$ \\
\hline-0.2999 & -0.6219 & -1.6810 & 5.0000 & 0.3792 & 1.3061 & 0.0070 \\
\hline
\end{tabular}

Minimize

$$
\begin{aligned}
E[\text { Total Cost }]=- & 0.4 \sigma_{t} \delta-500 \rho_{y t}^{2} \sigma_{y}^{2} \delta \varphi(\delta) \\
+ & \left\{500 \sigma_{y}^{2}\left(1-\rho_{y t}^{2}\right)+500 \rho_{y t}^{2} \sigma_{y}^{2}-160\right\} \\
& \times \Phi(\delta)+260-250 \sigma_{y}^{2}
\end{aligned}
$$

\section{Closed-Form Solution}

$$
\begin{aligned}
& \delta^{*}= \pm(-2 \text { Lambert } W \\
& \times\left(\frac{-0.2 \sigma_{1} \sqrt{2 \pi} e^{\left(\left(160-500 \sigma_{y}^{2}\left(1-\rho_{y t}^{2}\right)\right) / 1000 \rho_{y t}^{2} \sigma_{y}^{2}\right)}}{500 \rho_{y t}^{2} \sigma_{y}^{2}}\right) \\
&\left.+\left(\frac{160-500 \sigma_{y}^{2}\left(1-\rho_{y t}^{2}\right)}{500 \rho_{y t}^{2} \sigma_{y}^{2}}\right)\right)^{1 / 2} \\
&=0.8910
\end{aligned}
$$

$\mathrm{USL}^{*}=1.3806$ and $\mathrm{LSL}^{*}=1.2317$.

The optimal tolerance $\delta^{*}$ is 0.8910 and then $\mathrm{USL}^{*}$ and LSL $^{*}$ are 1.3806 and 1.2317, respectively. Figure 5 illustrates the relationship between $\delta$ and all relevant costs which are expected total cost, expected manufacturing cost, cost incurred by the expected quality loss, and expected rejection cost including expected rework cost and expected scrap cost.

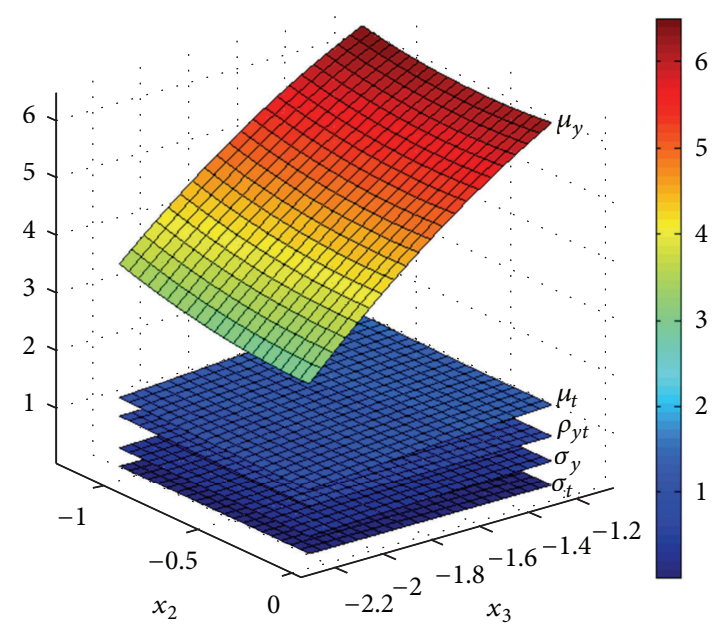

Figure 2: Plot of $\widehat{\mu}_{y}, \widehat{\sigma}_{y}^{2}, \widehat{\mu}_{t}, \widehat{\sigma}_{t}^{2}$, and $\widehat{\rho}_{y t}$ with respect to $x_{1}^{*}$.

In order to verify the optimality conditions of $\delta^{*}$, the plots of the first and the second derivatives of the expected total cost function with respect to $\delta$ are provided in Figures 6 and 7, respectively. The former shows that, at $\delta^{*}$, the value of $\partial E$ [Total Cost] $/ \partial \delta$ is zero; while the latter shows that, at $\delta^{*}$, the expected total cost is minimum because the value of $\partial^{2} E$ [Total Cost $] / \partial \delta^{2}$ is greater than zero. Also, the value of $\delta^{*}$ is between 0 and 1.6712, which is relevant to the interval defined in (25).

Three sensitivity analyses were conducted in order to determine the impact of $\theta, C_{\text {rework }}$, and $C_{\text {scrap }}$ on 


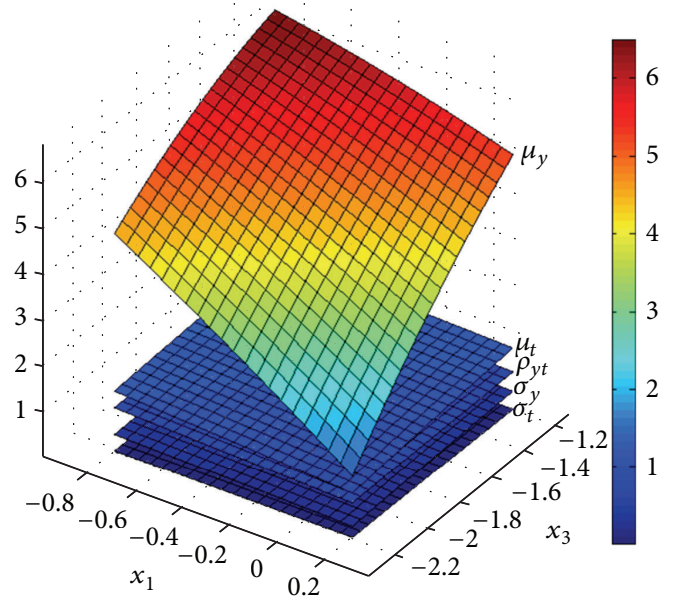

FIGURE 3: Plot of $\widehat{\mu}_{y}, \widehat{\sigma}_{y}^{2}, \widehat{\mu}_{t}, \widehat{\sigma}_{t}^{2}$, and $\widehat{\rho}_{y t}$ with respect to $x_{2}^{*}$.

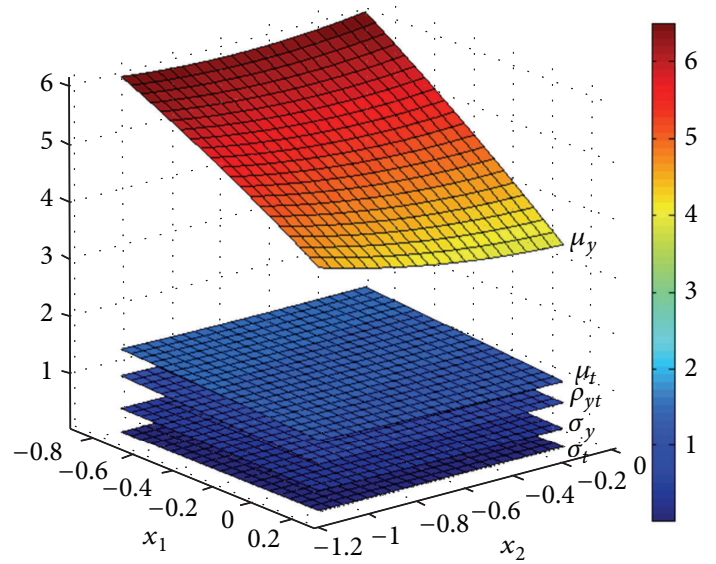

FIgURE 4: Plot of $\widehat{\mu}_{y}, \widehat{\sigma}_{y}^{2}, \widehat{\mu}_{t}, \widehat{\sigma}_{t}^{2}$, and $\widehat{\rho}_{y t}$ with respect to $x_{3}^{*}$.

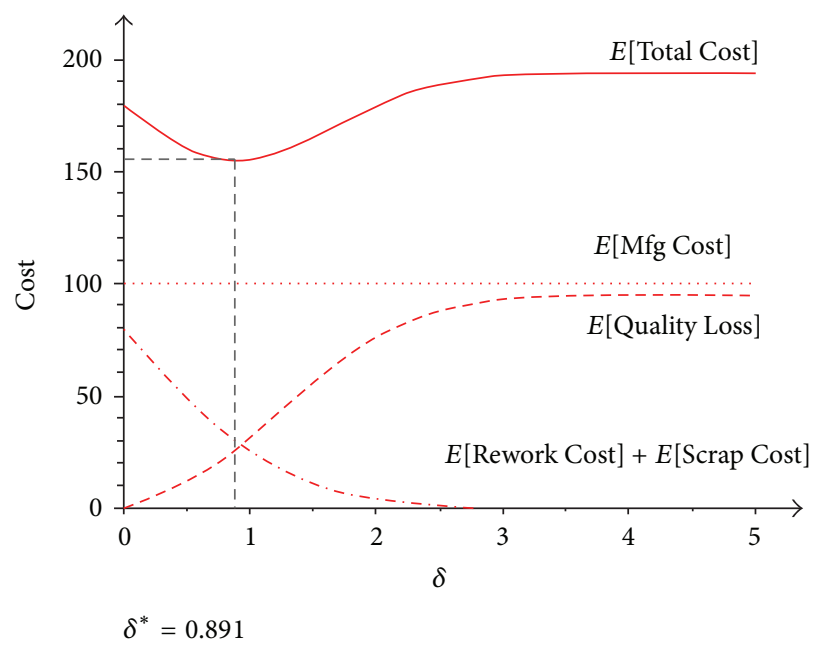

Figure 5: Plots of $E$ [Total Cost], $E$ [Mfg Cost], $E$ [Quality Loss], and $E[$ Rework Cost $]+E$ [Scrap Cost] with respect to $\delta$.

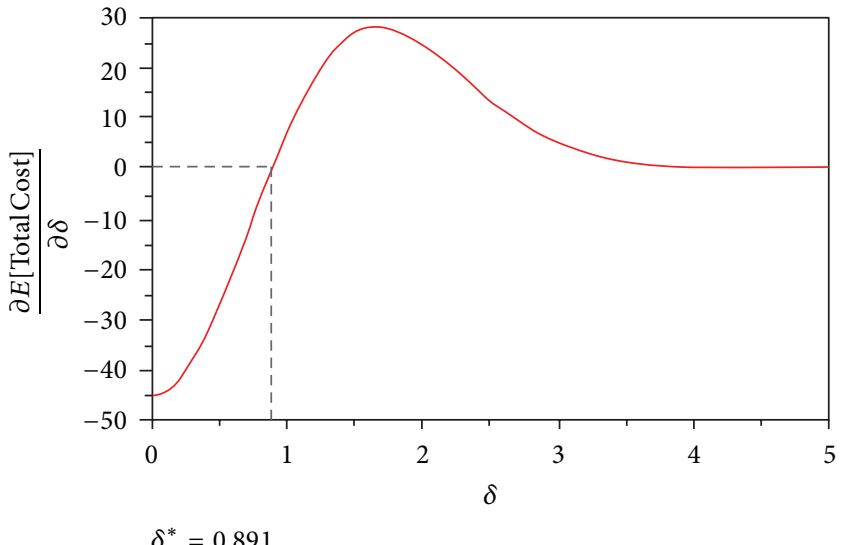

FIGURE 6: Plot of $\partial E[$ Total Cost $] / \partial \delta$ with respect to $\delta$.

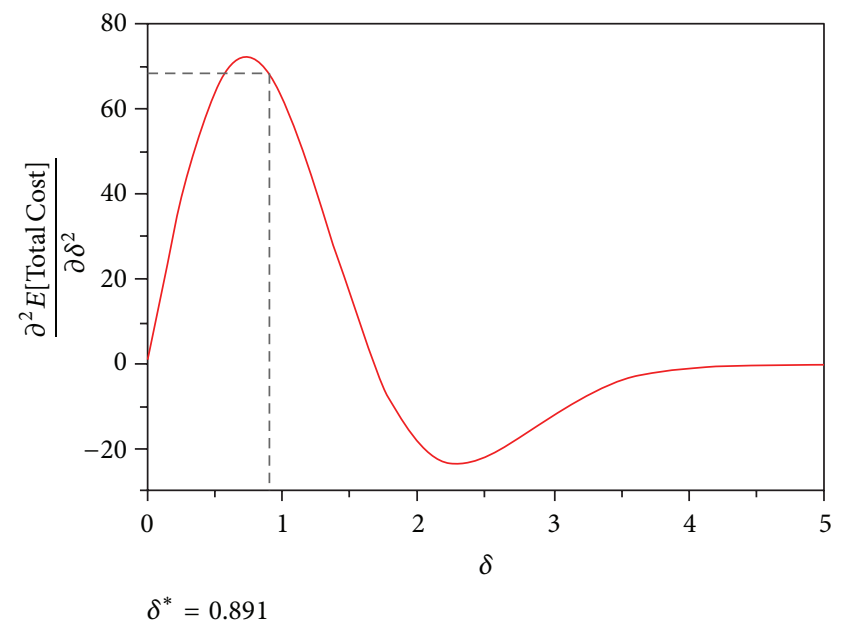

Figure 7: Plot of $\partial^{2} E[$ Total Cost $] / \partial \delta^{2}$ with respect to $\delta$.

$E$ [Rework Cost] around $\delta^{*}$ A full data set of each sensitivity analysis is provided in Tables 9, 10, and 11, respectively. First is the effect of $\theta$ while $C_{\text {rework }}$ and $C_{\text {scrap }}$ are held at 80 . Figure 8 identifies that while the value of $\theta$ changes from 100 to 400 with an increment of 25 , the values of $E$ [Rework Cost], $E$ [Scrap Cost], $E[\mathrm{Mfg}$ Cost], and $E[$ Total Cost] increase; and while the value of $\delta$ decreases, $E$ [Quality Loss] has a concave pattern.

Second is the effect of $C_{\text {rework }}$ while $\theta$ and $C_{\text {scrap }}$ are held at 250 and 80 , respectively. Figure 9 demonstrates that while the value of $C_{\text {rework }}$ changes from 40 to 160 with an increment of 8, the values of $\delta^{*}, E$ [Rework Cost], $E$ [Quality Loss], and $E$ [Total Cost] increase; and while the values of $E$ [Scrap Cost] and $E$ [Mfg Cost] decrease, the rejection cost (which is a summation of $E$ [Rework Cost] and $E$ [Scrap Cost]) has a concave pattern.

Last is the effect of $C_{\text {scrap }}$ while $\theta$ and $C_{\text {rework }}$ are held at 250 and 80 , respectively. Figure 10 represents that while the value of $C_{\text {scrap }}$ changes from 40 to 160 with an increment of 8 , the values of, $E[$ Scrap Cost], E[Quality Loss], and 


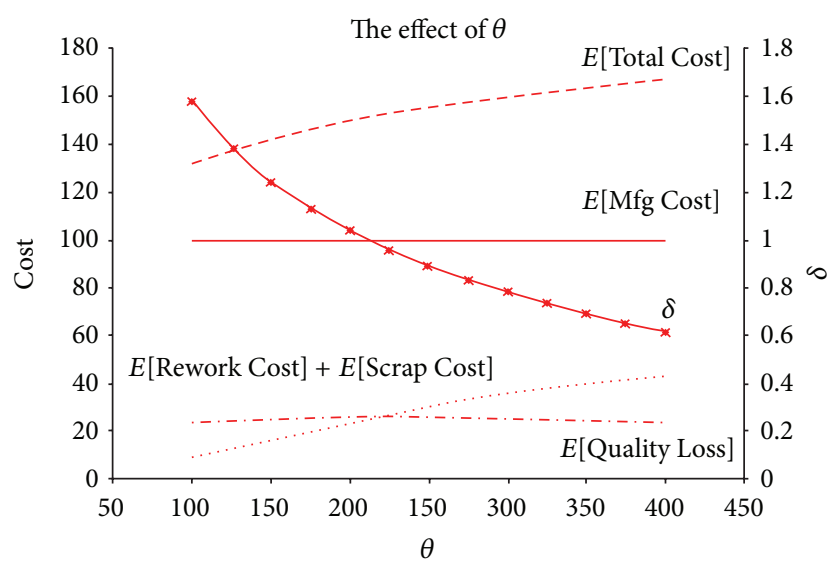

FIgURE 8: The effect of $\theta$ on minimum costs $\left(C_{\text {rework }}=80\right.$ and $C_{\text {scrap }}=$ $80)$.

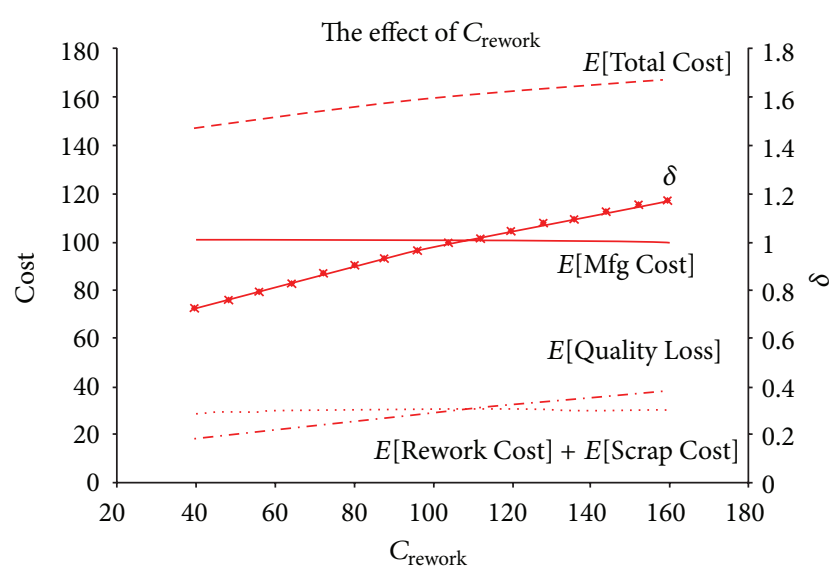

FIGURE 9: The effect of $C_{\text {rework }}$ on minimum $\operatorname{costs}\left(\theta=250\right.$ and $C_{\text {scrap }}$ $=80$ ).

$E$ [Total Cost] increase. Similar to the second sensitivity analysis, while the values of $E$ [Rework Cost] and $E$ [Mfg Cost] decrease, the rejection cost has a concave pattern.

\section{Conclusions and Future Research}

This paper proposed the optimization schemes for determining an optimal factor setting and tolerances for a destructive pharmaceutical characteristic. In reality, there are a number of destructive quality characteristics in pharmaceutical processes. Conceptualizing destructive pharmaceutical characteristics for the purpose of optimizing such pharmaceutical parameters, to our best knowledge, might be the first attempt in this field. To find the optimal factor setting with minimal variability, $\mathrm{RD}$ is an effective method. It is more effective when applied in the early stage of process design. Then, to remove possible variability, TD based on the concept of Taguchi's offline quality management is applied. With the result from $\mathrm{RD}$ stage, optimal tolerance and specification limits are obtained.

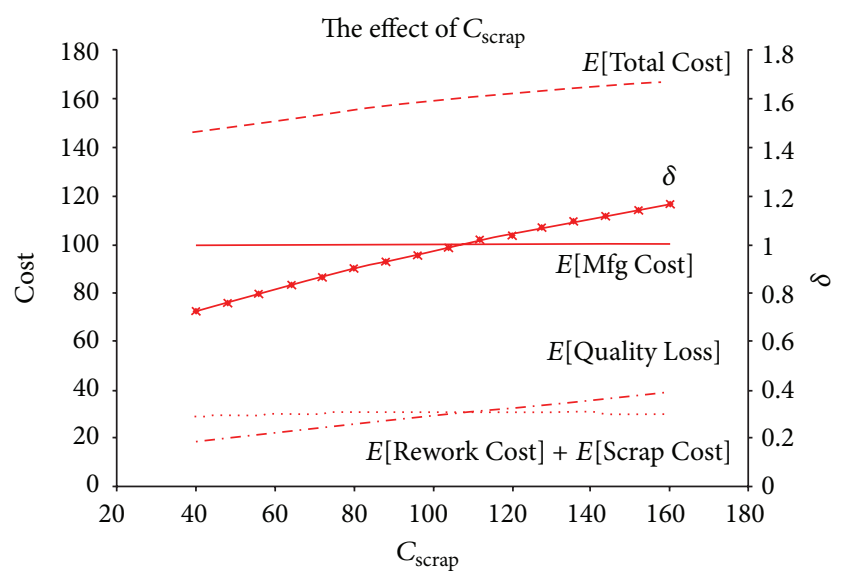

FIgURE 10: The effect of $C_{\text {scrap }}$ on minimum costs $(\theta=250$ and $\left.C_{\text {rework }}=80\right)$.

The proposed approach integrated the RD and TD concepts with the use of RSM and surrogate variables within the framework of destructive characteristics in order to achieve the target requirement with minimal variability and handle the destructive quality characteristic under $100 \%$ inspection. The experimental design format to find the relationship between a destructive quality characteristic and its associated surrogate variable was also proposed. Moreover, mathematical procedures for finding optimal tolerance and its closed-form solution by applying the Lambert $W$ function principle were provided. The numerical example showed that the proposed approach not only delivered a robust factor setting but also considered tradeoff between the level of quality and cost.

Based on the results of this paper, a significant extension to support multiple quality characteristics can be a potential future research topic. In addition, current interesting issues, related to fractals associated with fractal dimension and Hurst parameter [52-58] in the field of pharmaceutical science and technology, can be incorporated into the proposed robust-tolerance design optimization method. By observing the fractal phenomena, these possible further approaches may achieve the realization of process understanding (PU) and process analysis technology (PAT) in pharmaceutical processes.

\section{Appendices}

\section{A. Sensitivity Analysis}

This section provides a result of the sensitivity analyses conducted for the numerical example in Section 4. Table 9 shows effects of $\theta$ while holding $C_{\text {rework }}$ and $C_{\text {scrap }}$ at 80 and while changing values of $\theta$ from 100 to 400 with an increment of 25. Table 10 shows effects of $C_{\text {rework }}$ while holding $\theta$ and $C_{\text {scrap }}$ at 250 and 80 , respectively and while changing values of $C_{\text {rework }}$ from 40 to 160 with an increment of 8 . Table 11 shows effects of $C_{\text {scrap }}$ while holding $\theta$ and $C_{\text {rework }}$ at 250 and 80, respectively and while changing values of $C_{\text {scrap }}$ from 40 to 160 with an increment of 8 . 
TABle 9: Effects of $\theta$ on minimum costs $\left(C_{\text {rework }}=80\right.$ and $\left.C_{\text {scrap }}=80\right)$.

\begin{tabular}{lcccccc}
\hline$\theta$ & $\delta^{*}$ & $E[$ Rework Cost] & $E$ [Scrap Cost] & $E$ [Quality Loss] & $E$ [Mfg Cost] & $E$ [Total Cost] \\
\hline 100 & 1.5737 & 4.6227 & 4.6227 & 23.1282 & 99.9474 & 132.3210 \\
125 & 1.3838 & 6.6567 & 6.6567 & 24.3612 & 99.9537 & 137.6283 \\
150 & 1.2414 & 8.5787 & 8.5787 & 25.0187 & 99.9585 & 142.1345 \\
175 & 1.1288 & 10.3601 & 10.3601 & 25.3359 & 99.9623 & 146.0184 \\
200 & 1.0363 & 12.0022 & 12.0022 & 25.4404 & 99.9653 & 149.4102 \\
225 & 0.9583 & 13.5167 & 13.5167 & 25.4044 & 99.9680 & 152.4057 \\
250 & 0.8910 & 14.9177 & 14.9177 & 25.2706 & 99.9702 & 155.0762 \\
275 & 0.8319 & 16.2194 & 16.2194 & 25.0646 & 99.9722 & 157.4755 \\
300 & 0.7792 & 17.4346 & 17.4346 & 24.8024 & 99.9739 & 159.6456 \\
325 & 0.7317 & 18.5747 & 18.5747 & 24.4940 & 99.9755 & 161.6189 \\
350 & 0.6883 & 19.6496 & 19.6496 & 24.1454 & 99.9770 & 163.4216 \\
375 & 0.6484 & 20.6679 & 20.6679 & 23.7604 & 99.9783 & 165.0745 \\
400 & 0.6114 & 21.6372 & 21.6372 & 23.3408 & 99.9796 & 166.5948 \\
\hline
\end{tabular}

TABLE 10: Effects of $C_{\text {rework }}$ on minimum costs $\left(\theta=250\right.$ and $\left.C_{\text {scrap }}=80\right)$.

\begin{tabular}{lcccccc}
\hline$C_{\text {rework }}$ & $\delta^{*}$ & $E$ [Rework Cost] & $E$ [Scrap Cost] & $E$ [Quality Loss] & $E$ [Mfg Cost] & $E$ [Total Cost] \\
\hline 40 & 0.7169 & 9.4683 & 18.9365 & 18.2914 & 99.9760 & 146.6723 \\
48 & 0.7550 & 10.8066 & 18.0109 & 19.7268 & 99.9748 & 148.5190 \\
56 & 0.7912 & 12.0078 & 17.1541 & 21.1413 & 99.9735 & 150.2767 \\
64 & 0.8258 & 13.0859 & 16.3573 & 22.5362 & 99.9724 & 151.9518 \\
72 & 0.8590 & 14.0524 & 15.6138 & 23.9125 & 99.9713 & 153.5500 \\
80 & 0.8910 & 14.9177 & 14.9177 & 25.2706 & 99.9702 & 155.0762 \\
88 & 0.9219 & 15.6907 & 14.2643 & 26.6108 & 99.9692 & 156.5349 \\
96 & 0.9517 & 16.3794 & 13.6495 & 27.9333 & 99.9682 & 157.9303 \\
104 & 0.9807 & 16.9908 & 13.0699 & 29.2381 & 99.9672 & 159.2660 \\
112 & 1.0088 & 17.5314 & 12.5224 & 30.5253 & 99.9663 & 160.5454 \\
120 & 1.0362 & 18.0068 & 12.0045 & 31.7949 & 99.9653 & 161.7715 \\
128 & 1.0629 & 18.4222 & 11.5139 & 33.0467 & 99.9645 & 162.9472 \\
136 & 1.0889 & 18.7823 & 11.0484 & 34.2808 & 99.9636 & 164.0751 \\
144 & 1.1143 & 19.0915 & 10.6064 & 35.4970 & 99.9627 & 165.1576 \\
152 & 1.1391 & 19.3537 & 10.1861 & 36.6954 & 99.9619 & 166.1971 \\
160 & 1.1634 & 19.5724 & 9.7862 & 37.8758 & 99.9611 & 167.1955 \\
\hline
\end{tabular}

\section{B. The Lambert $W$ Function}

Since the Lambert $W$ function is defined as $f(\Psi)=\Psi e^{\Psi}$, Lambert $W(\Psi)$ allows solving such functional equation as $g(\Psi) e^{g(\Psi)}=\Psi$ and $g(\Psi)=e^{\text {Lambert } W(\ln (\Psi))}$, and $\Psi e^{\Psi}=\omega$ and $\Psi=$ Lambert $W(\omega)([20,53])$. As shown in Section 3.3, this functional equation is encountered in the tolerance optimization models involving normally distributed random variables and use of the Lambert $W$ function facilitates obtaining a closed-form solution for the optimal specification limits. Based on Lambert $W$ principle [52], Shin et al. [20] consider the equation as follows:

$$
\Delta_{4}=\Delta_{1}\left(x+\Delta_{2}\right) e^{\Delta_{3} x} .
$$

By dividing $\Delta_{1}$, multiplying $\Delta_{3}$, and denoting $\Psi=\Delta_{3}(x+$ $\left.\Delta_{2}\right)$, (B.1) becomes

$$
\frac{\Delta_{3} \Delta_{4} e^{\Delta_{2} \Delta_{3}}}{\Delta_{1}}=\Psi e^{\Psi_{1}} .
$$

Replacing $\Delta_{3} \Delta_{4} e^{\Delta_{2} \Delta_{3}} / \Delta_{1}$ by $\omega$, (B.2) then becomes

$$
\Psi=\text { Lambert } W(\omega) .
$$

Denoting $\omega=\Delta_{3} \Delta_{4} e^{\Delta_{2} \Delta_{3}} / \Delta_{1}$, (B.3) can then be

$$
\Psi=\text { Lambert } W\left(\frac{\Delta_{3} \Delta_{4} e^{\Delta_{2} \Delta_{3}}}{\Delta_{1}}\right) .
$$


TABLE 11: Effects of $C_{\text {scrap }}$ on minimum costs $\left(\theta=250\right.$ and $\left.C_{\text {rework }}=80\right)$.

\begin{tabular}{lcccccc}
\hline$C_{\text {scrap }}$ & $\delta^{*}$ & $E[$ Rework Cost] & $E$ [Scrap Cost] & $E$ [Quality Loss] & $E$ [Mfg Cost] & $E$ [Total Cost] \\
\hline 40 & 0.7169 & 18.9365 & 9.4683 & 18.2914 & 99.9760 & 146.6723 \\
48 & 0.7550 & 18.0109 & 10.8066 & 19.7268 & 99.9748 & 148.5190 \\
56 & 0.7912 & 17.1541 & 12.0078 & 21.1413 & 99.9735 & 150.2767 \\
64 & 0.8258 & 16.3573 & 13.0859 & 22.5362 & 99.9724 & 151.9518 \\
72 & 0.8590 & 15.6138 & 14.0524 & 23.9125 & 99.9713 & 153.5500 \\
80 & 0.8910 & 14.9177 & 14.9177 & 25.2706 & 99.9702 & 155.0762 \\
88 & 0.9219 & 14.2643 & 15.6907 & 26.6108 & 99.9692 & 156.5349 \\
96 & 0.9517 & 13.6495 & 16.3794 & 27.9333 & 99.9682 & 157.9303 \\
104 & 0.9807 & 13.0699 & 16.9908 & 29.2381 & 99.9672 & 159.2660 \\
112 & 1.0088 & 12.5224 & 17.5314 & 30.5253 & 99.9663 & 160.5454 \\
120 & 1.0362 & 12.0045 & 18.0068 & 31.7949 & 99.9653 & 161.7715 \\
128 & 1.0629 & 11.5139 & 18.4222 & 33.0467 & 99.9645 & 162.9472 \\
136 & 1.0889 & 11.0484 & 18.7823 & 34.2808 & 99.9636 & 164.0751 \\
144 & 1.1143 & 10.6064 & 19.0915 & 35.4970 & 99.9627 & 165.1576 \\
152 & 1.1391 & 10.1861 & 19.3537 & 36.6954 & 99.9619 & 166.1971 \\
160 & 1.1634 & 9.7862 & 19.5724 & 37.8758 & 99.9611 & 167.1955 \\
\hline
\end{tabular}

Substituting $\Psi$ to $\Delta_{3}\left(x+\Delta_{2}\right)$, the solution in terms of $x$ can then be obtained as follows:

$$
x= \pm \sqrt{\left\{\frac{\text { Lambert } W\left(\Delta_{3} \Delta_{4} e^{\Delta_{2} \Delta_{3}} / \Delta_{1}\right)}{\Delta_{3}}\right\}-\Delta_{2} .}
$$

\section{Acknowledgment}

This work was supported by the Dong-A University research fund.

\section{References}

[1] G. Taguchi, Introduction to Quality Engineering: Designing Quality into Products and Processes, Asian Productivity Association, Tokyo, Japan, 1986.

[2] G. Taguchi, Taguchi on Robust Technology Development: Bringing Quality Engineering Upstream, American Society of Mechanical Engineers, New York, NY, USA, 1993.

[3] S. Shin and B. R. Cho, "Bias-specified robust design optimization and its analytical solutions," Computers \& Industrial Engineering, vol. 48, no. 1, pp. 129-140, 2005.

[4] G. Box, "Signal-to-noise ratios, performance criteria, and transformations," Technometrics, vol. 30, no. 1, pp. 1-40, 1988.

[5] G. Box, S. Bisgaard, and C. Fung, "An explanation and critique of Taguchi's contribution to quality engineering," Quality and Reliability Engineering International, vol. 4, no. 2, pp. 123-131, 1988.

[6] R. V. León, A. C. Shoemaker, and R. N. Kacker, "Performance measures independent of adjustment. An explanation and extension of Taguchi's signal-to-noise ratios," Technometrics, vol. 29, no. 3, pp. 253-285, 1987.

[7] V. N. Nair, "Taguchi's parameter design: a panel discussion," Technometrics, vol. 34, no. 2, pp. 127-161, 1992.

[8] K. L. Tsui, "An overview of Taguchi method and newly developed statistical methods for robust design," IIE Transactions, vol. 24 , no. 5 , pp. $44-57,1992$.
[9] G. G. Vining and R. H. Myers, "Combining Taguchi and response surface philosophies: a dual response approach," Journal of Quality Technology, vol. 22, no. 1, pp. 38-45, 1990.

[10] K. A. F. Copeland and P. R. Nelson, "Dual response optimization via direct function minimization," Journal of Quality Technology, vol. 28, no. 3, pp. 331-336, 1996.

[11] E. Del Castillo and D. C. Montgomery, "A nonlinear programming solution to the dual response problem," Journal of Quality Technology, vol. 25, no. 3, pp. 199-204, 1993.

[12] K. J. Kim and D. K. J. Lin, "Dual response surface optimization: a fuzzy modeling approach," Journal of Quality Technology, vol. 30, no. 1, pp. 1-10, 1998.

[13] Y. J. Kim and B. R. Cho, "Development of priority-based robust design," Quality Engineering, vol. 14, no. 3, pp. 355-363, 2002.

[14] O. Köksoy and N. Doganaksoy, "Joint optimization of mean and standard deviation using response surface methods," Journal of Quality Technology, vol. 35, no. 3, pp. 239-252, 2003.

[15] S. B. Lee, C. Park, and B. R. Cho, "Development of a highly efficient and resistant robust design," International Journal of Production Research, vol. 45, no. 1, pp. 157-167, 2007.

[16] D. K. J. Lin and W. Tu, "Dual response surface optimization," Journal of Quality Technology, vol. 27, no. 1, pp. 34-39, 1995.

[17] K. Tang and J. Tang, "Design of product specifications for multicharacteristic inspection," Management Science, vol. 35, no. 6, pp. 743-756, 1989.

[18] B. R. Cho, Optimization Issue in Quality Engineering, University of Oklahoma, Norman, Okla, USA, 1994.

[19] L. C. Tang and K. Xu, "A unified approach for dual response surface optimization," Journal of Quality Technology, vol. 34, no. 4, pp. 437-447, 2002.

[20] S. Shin, M. S. Govindaluri, and B. R. Cho, "Integrating the Lambert W function to a tolerance optimization problem," Quality and Reliability Engineering International, vol. 21, no. 8, pp. 795-808, 2005.

[21] K. W. Chase, W. H. Greenwood, B. G. Loosli, and L. F. Hauglund, "Least cost tolerance allocation for mechanical assemblies with automated process selection," Manufacturing review, vol. 3, no. 1, pp. 49-59, 1990. 
[22] Y. J. Kim and B. R. Cho, "The use of response surface designs in the selection of optimum tolerance allocation," Quality Engineering, vol. 13, no. 1, pp. 35-42, 2000.

[23] F. H. Speckhart, "Calculation of tolerance based on a minimum cost approach," Journal of Engineering for Industry-Transactions of the ASME, vol. 94, no. 2, pp. 447-453, 1972.

[24] M. F. Spotts, "Allocation of tolerances to minimize cost of assembly," Journal of Engineering For Industry-Transactions of the ASME, vol. 95, no. 3, pp. 762-764, 1973.

[25] Y. Fathi, "Producer-consumer tolerances," Journal of Quality Technology, vol. 22, no. 2, pp. 138-145, 1990.

[26] Y. J. Kim and B. R. Cho, "Economic integration of design optimization," Quality Engineering, vol. 12, no. 4, pp. 561-567, 2000.

[27] M. D. Phillips and B. R. Cho, "An empirical approach to designing product specifications: a case study," Quality Engineering, vol. 11, no. 1, pp. 91-100, 1999.

[28] K. Tang, "Economic design of product specifications for a complete inspection plan," International Journal of Production Research, vol. 26, no. 2, pp. 203-217, 1988.

[29] A. Jeang, "An approach of tolerance design for quality improvement and cost reduction," International Journal of Production Research, vol. 35, no. 5, pp. 1193-1211, 1997.

[30] P. Wang and M. Liang, "An integrated approach to tolerance synthesis, process selection and machining parameter optimization problems," International Journal of Production Research, vol. 43, no. 11, pp. 2237-2262, 2005.

[31] S. Shin and B. R. Cho, "Integrating a bi-objective paradigm to tolerance optimization," International Journal of Production Research, vol. 45, no. 23, pp. 5509-5525, 2007.

[32] H. P. Peng, X. Q. Jiang, and X. J. Liu, "Concurrent optimal allocation of design and process tolerances for mechanical assemblies with interrelated dimension chains," International Journal of Production Research, vol. 46, no. 24, pp. 6963-6979, 2008.

[33] G. Prabhaharan, R. Ramesh, and P. Asokan, "Concurrent optimization of assembly tolerances for quality with position control using scatter search approach," International Journal of Production Research, vol. 45, no. 21, pp. 4959-4988, 2007.

[34] D. S. Bai and S. H. Hong, "Economic screening procedures using a correlated variable with multidecision alternatives," Naval Research Logistics, vol. 39, no. 4, pp. 471-485, 1992.

[35] D. S. Bai, S. B. Kim, and M. C. Riew, "Economic screening procedures based on correlated variables," Metrika, vol. 37, no. 5, pp. 263-280, 1990.

[36] R. J. Boys and I. R. Dunsmore, "Screening in a normal model," Journal of the Royal Statistical Society B, vol. 48, no. 1, pp. 60-69, 1986.

[37] S. H. Hong, M. K. Lee, H. M. Kwon, and S. B. Kim, "A continuous screening procedure using the performance and surrogate variables," International Journal of Production Research, vol. 39, no. 11, pp. 2333-2340, 2001.

[38] S. B. Kim and D. S. Bai, "Economic design of one-sided screening procedures based on a correlated variable with all parameters unknown," Metrika, vol. 39, no. 2, pp. 85-93, 1992.

[39] H. M. Kwon, S. H. Hong, M. K. Lee, and S. B. Kim, "A process monitoring procedure based on a surrogate variable for dichotomous performance variable," IIE Transactions, vol. 33, no. 12, pp. 1129-1133, 2001.

[40] M. K. Lee and E. A. Elsayed, "Process mean and screening limits for filling processes under two-stage screening procedure,"
European Journal of Operational Research, vol. 138, no. 1, pp. 118126, 2002.

[41] H. Moskowitz, R. Plante, and H. T. Tsai, "Single-sided economic screening models incorporating individual unit misclassification error and risk preference," European Journal of Operational Research, vol. 53, no. 2, pp. 228-243, 1991.

[42] H. Moskowitz, R. Plante, and H. T. Tsai, "A multistage screening model for evaluation and control of misclassification error in the detection of hypertension," Management Science, vol. 39, no. 3, pp. 307-321, 1993.

[43] K. Tang, "Economic design of a one-sided screening procedure using a correlated variable," Technometrics, vol. 29, no. 4, pp. 477-485, 1987.

[44] H. T. Tsai, H. Moskowitz, and J. Tang, "One-sided single screening procedure based on individual unit misclassification error," IIE Transactions, vol. 27, no. 6, pp. 695-706, 1995.

[45] Q. Wang, "Dimension reduction in partly linear error-inresponse models with validation data," Journal of Multivariate Analysis, vol. 85, no. 2, pp. 234-252, 2003.

[46] S.-F. Wu and M.-Y. Cheng, "A two-sided sequential screening procedure based on individual misclassification error," Computational Statistics \& Data Analysis, vol. 40, no. 2, pp. 375-391, 2002.

[47] S. Shin, N. K. V. Truong, B. R. Cho, and S. H. Hong, "Development of a sequential robust-tolerance design model for a destructive quality characteristic," Computers \& Industrial Engineering, vol. 60, no. 4, pp. 777-789, 2011.

[48] A. M. Patel, "Computer-aided assignment of tolerances," in Proceedings of the 17th Design and Automation, pp. 129-133, ACM, New York, NY, USA, 1980.

[49] O. Bjorke, Computer Aided Tolerancing, American Society of Mechanical Engineers, New York, NY, USA, 2nd edition, 1989.

[50] K. W. Chase, W. H. Greenwood, B. G. Loosli, and L. F. Hauglund, "Least cost tolerance allocation for mechanical assemblies with automated process selection," Manufacturing review, vol. 3, no. 1, pp. 49-59, 1990.

[51] TeraView, "Non-destructive hardness testing and identification of potential capping in tablets using terahertz pulsed spectroscopy and imaging," 2008, http://www.teraview.com/ .

[52] G. Valsami and P. Macheras, "Determination of fractal reaction dimension in dissolution studies," European Journal of Pharmaceutical Sciences, vol. 3, no. 3, pp. 163-169, 1995.

[53] J. Akimoto, K. Takayama, K. Isowa, and T. Nagai, "Evaluation of skin irritation of percutaneous absorption promoters by means of fractal dimension of rat skin structure," International Journal of Pharmaceutics, vol. 128, no. 1-2, pp. 251-260, 1996.

[54] M. A. Holgado, M. J. Fernández-Hervás, J. Alvarez-Fuentes, M. T. Vela, A. M. Rabasco, and A. Fini, "Characterization of modified paracetamol by means of SEM and fractal analysis," International Journal of Pharmaceutics, vol. 142, no. 2, pp. 143151, 1996.

[55] N. Pippa, S. Pispas, and C. Demetzos, “The fractal hologram and elucidation of the structure of liposomal carriers in aqueous and biological media," International Journal of Pharmaceutics, vol. 430, no. 1-2, pp. 65-73, 2012.

[56] M. Li and W. Zhao, "On $1 / f$ noise," Mathematical Problems in Engineering, vol. 2012, Article ID 673648, 22 pages, 2012.

[57] M. Li and W. Zhao, "Visiting power laws in cyber-physical networking systems," Mathematical Problems in Engineering, vol. 2012, Article ID 302786, 13 pages, 2012. 
[58] M. Li, Y. Q. Chen, J. Y. Li, and W. Zhao, "Hölder scales of sea level," Mathematical Problems in Engineering, vol. 2012, Article ID 863707, 22 pages, 2012. 


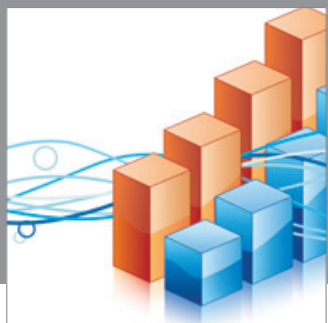

Advances in

Operations Research

mansans

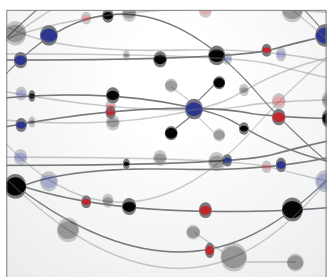

The Scientific World Journal
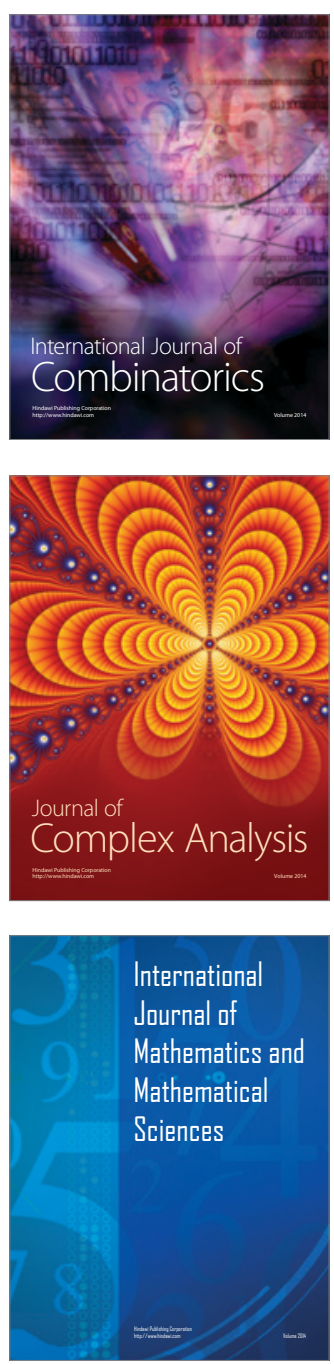
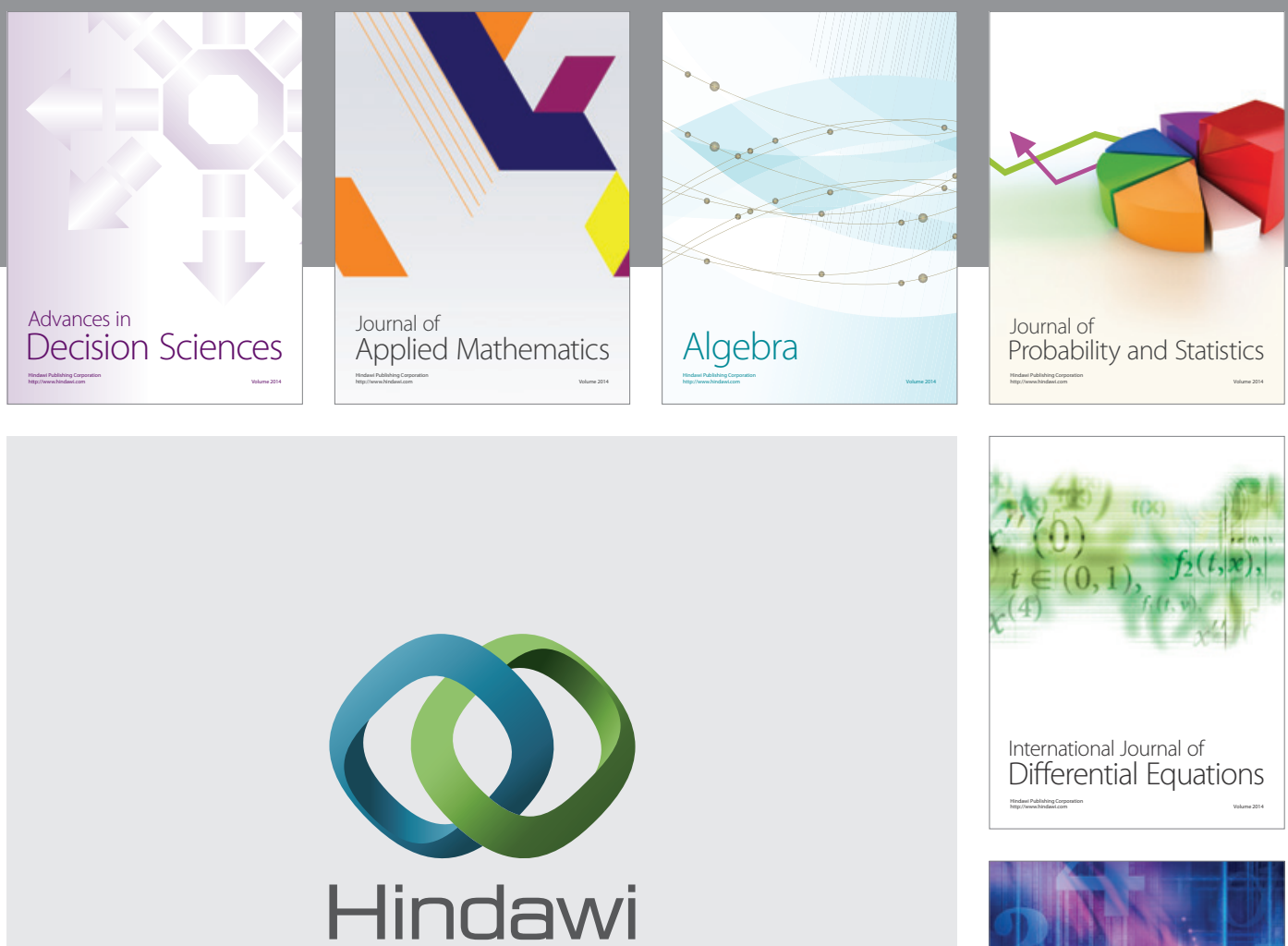

Submit your manuscripts at http://www.hindawi.com
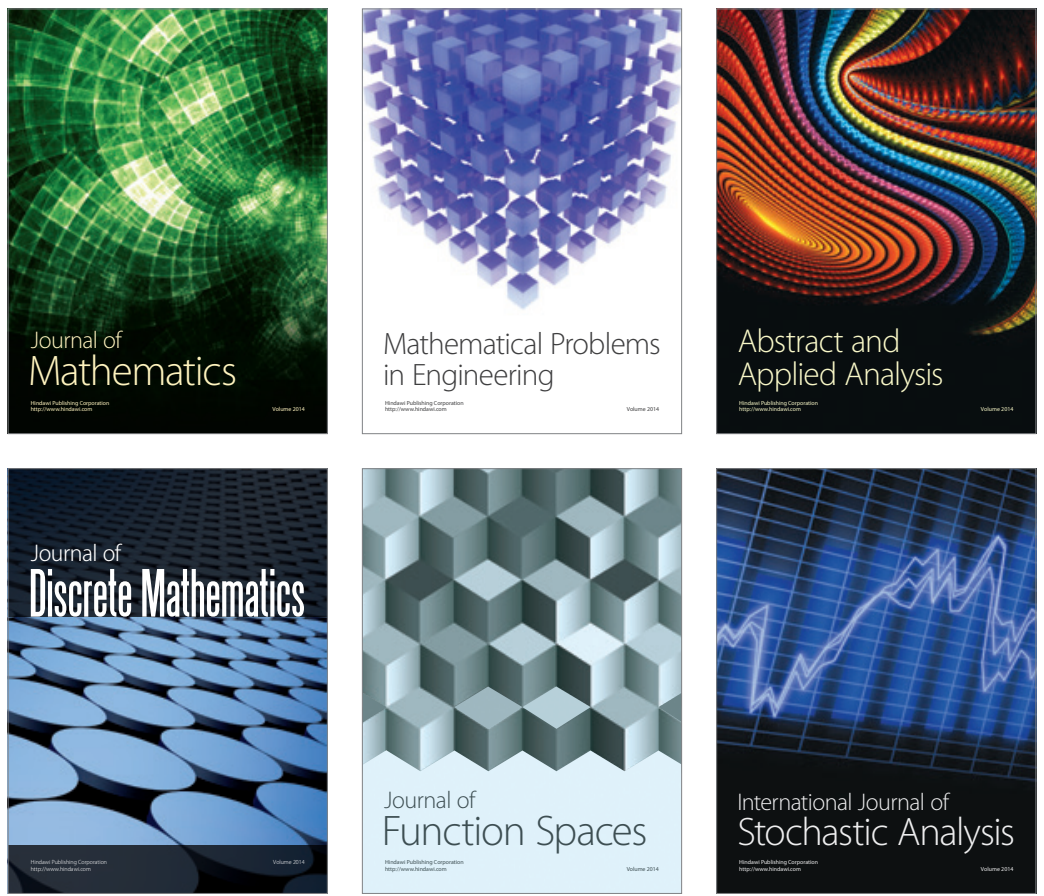

Journal of

Function Spaces

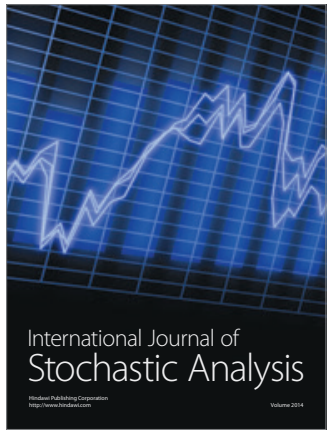

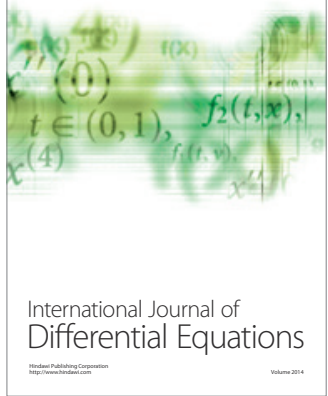
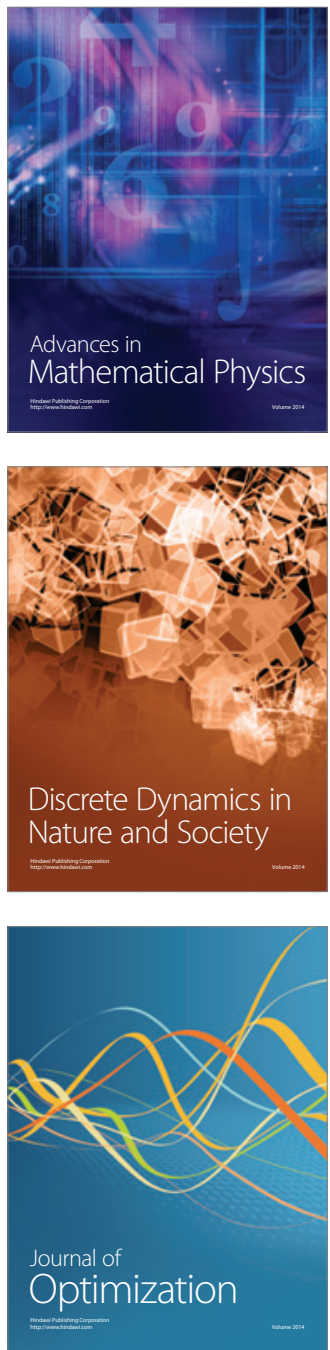